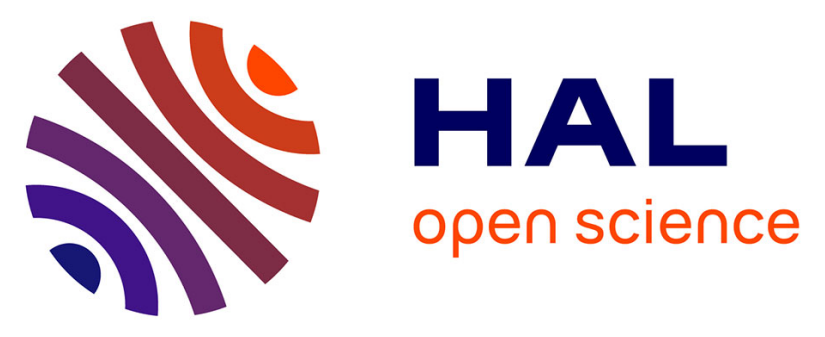

\title{
Nonlinear dynamics of two coupled bubbles oscillating inside a liquid-filled cavity surrounded by an elastic medium
}

\author{
Alexander A. Doinikov, Diane J Bienaimé, S. Roberto Gonzalez-Avila, \\ Claus-Dieter Ohl, Philippe Marmottant
}

\section{To cite this version:}

Alexander A. Doinikov, Diane J Bienaimé, S. Roberto Gonzalez-Avila, Claus-Dieter Ohl, Philippe Marmottant. Nonlinear dynamics of two coupled bubbles oscillating inside a liquid-filled cavity surrounded by an elastic medium. Physical Review E , 2019, 99 (5), 10.1103/PhysRevE.99.053106 . hal-02402114

\section{HAL Id: hal-02402114 \\ https://hal.science/hal-02402114}

Submitted on 13 Dec 2019

HAL is a multi-disciplinary open access archive for the deposit and dissemination of scientific research documents, whether they are published or not. The documents may come from teaching and research institutions in France or abroad, or from public or private research centers.
L'archive ouverte pluridisciplinaire HAL, est destinée au dépôt et à la diffusion de documents scientifiques de niveau recherche, publiés ou non, émanant des établissements d'enseignement et de recherche français ou étrangers, des laboratoires publics ou privés. 


\title{
Nonlinear dynamics of two coupled bubbles oscillating inside a liquid-filled cavity surrounded by an elastic medium
}

\author{
Alexander A. Doinikov, ${ }^{1}$ Diane Bienaimé, ${ }^{1}$ S. Roberto Gonzalez-Avila, ${ }^{2}$ Claus- \\ Dieter Ohl, ${ }^{2}$ and Philippe Marmottant ${ }^{1}$ \\ ${ }^{1}$ LIPhy UMR 5588, CNRS/Université Grenoble-Alpes, Grenoble F-38401, France \\ ${ }^{2}$ Institute of Physics, Otto-von-Guericke-Universität Magdeburg, Universitätsplatz 2, 39016 \\ Magdeburg, Germany
}


A theory is developed to model the nonlinear dynamics of two coupled bubbles inside a spherical liquid-filled cavity surrounded by an elastic medium. The aim is to study how the conditions of full confinement affect the coupled oscillations of the bubbles. To make the problem amenable to analytical consideration, the bubbles are assumed to be located on a diameter of the cavity, which makes the problem axisymmetric. Equations for the pulsation and translation motion of the bubbles are derived by the Lagrangian formalism. The derived equations are used in numerical simulations. The behavior of two bubbles in a cavity is compared with the behavior of the same bubbles in an unbounded liquid. It is found that both forced and free oscillations of two bubbles in a cavity occur differently than those in an unbounded liquid. In particular, it is shown that the eigenfrequencies of a two-bubble system in a cavity are different from those in an unbounded liquid.

Key words: cavitation bubble, liquid-filled cavity, elastic confinement, eigenmode 


\section{INTRODUCTION}

Interest in bubble dynamics that occurs inside a liquid microinclusion enclosed in a solid medium is motivated by applications in geology [1,2], dynamics of porous media [3-5], biology [6-10], etc.

There are a number of theoretical studies on the dynamics of a single bubble in a spherical liquid-filled cavity surrounded by an infinite elastic solid [11-16]. In particular, it has been shown in Refs. [13], [14], and [16] that the finite-amplitude pulsation of a single bubble in a spherical cavity is governed by a Rayleigh-Plesset-like equation. There are differences in equations derived in these works, which result from different approximations that were used to model the behavior of the elastic surrounding medium. The most rigorous derivation was performed in Ref. [16]. The effect of confinement in all the equations is expressed in terms of the time-varying radius of the cavity. Therefore, all the equations reduce to the Rayleigh-Plesset equation when the cavity radius tends to infinity. It has been shown in Refs. [15] and [16] that the resonance frequency of the system (a cavity with a bubble inside) decreases with decreasing bubble radius, in contrast to what occurs in an unbounded liquid, where the resonance frequency of a bubble increases with decreasing bubble radius. This result is explained by the fact that the dynamics of the system is governed by the solid environment rather than by the bubble. This theoretical prediction is in agreement with experimental observations of Vincent et al. [12]. The dependence of the resonance frequency on the material parameters of the system, such as the modulus of rigidity of the solid, is not trivial and therefore cannot be described in brief. A detailed analysis of the behavior of the resonance frequency in various limiting cases was performed by Drysdale et al. [15]. As an interesting result, it should be mentioned that the resonance frequency does not become zero when the modulus of rigidity tends to infinity.

In nature and technology, the presence of more than one bubble in a cavity is a more general case than the presence of a single bubble. Therefore, a theoretical model that would describe the behavior of two coupled bubbles inside a cavity could be the next step in studying cavitation phenomena that occur under the conditions of microscopic confinement.

The dynamics of two interacting bubbles is a classical problem whose history goes back to the works of C. A. Bjerknes and his son V. F. K. Bjerknes [17]. Reviews on this problem can be found in Refs. [18-26]. The influence of two bubbles on the oscillations of each other is pointed out in the literature to lead to two main effects. First, the bubbles undergo attraction or repulsion 
depending on the value of the driving frequency with the respect to the natural frequencies of the bubbles. Second, the natural frequencies of the bubbles change in comparison with the natural frequencies that the bubbles have when they are separated in space. As a result, the acoustic response of two interacting bubbles is not just a sum of their individual responses even if the bubbles oscillate in the linear regime.

However, available theoretical studies on the dynamics of two interacting bubbles are devoted to bubbles in an unbounded liquid. To the best of our knowledge, there are no theoretical works on the dynamics of two interacting bubbles under the conditions of full confinement. Thus, the effect of full confinement on the behavior of interacting bubbles is presently unknown. This fact has served as a motivation for our study presented here.

Our study proposes a theory that describes the nonlinear dynamics of two interacting bubbles inside a spherical liquid-filled cavity surrounded by an elastic medium. The developed theory allows one to study the effect of full confinement on the coupled oscillations of the bubbles. In Sec. II, equations for the pulsation and translation motion of the bubbles are derived by the Lagrangian formalism. In Sec. III, the derived equations are used to perform numerical simulations and to compare results with the behavior of two interacting bubbles in an unbounded liquid.

\section{THEORY}

We assume that two spherical gas bubbles are inside a spherical liquid-filled cavity surrounded by an elastic solid layer; see Fig. 1. The bubble centers are assumed to be located on a diameter of the cavity. In this case, the problem possesses axial symmetry, which simplifies its analytical consideration. If this assumption is abandoned, the problem turns into a 3D problem and its solution becomes extremely complicated. We assume that, if the bubbles are not located along the diameter of the cavity, their behavior should demonstrate the same qualitative features, even though quantitative characteristics of this process will be different. Because, as we expect, the main physical factor that affects the bubble behavior in a cavity is the presence of full confinement as such. Therefore, we believe that our theoretical analysis, despite the fact that it is based on the simplified mathematical formulation, should give a correct insight into the dynamics of bubbles enclosed in a cavity.

We introduce a spherical coordinate system whose origin is at the center of the cavity and the $z$ axis goes through the bubble centers. The position vector $r$ in this coordinate system has the 
coordinates $(r, \theta, \varepsilon)$. Each bubble has its own local spherical coordinate system whose origin is at the moving center of the bubble. The position vector $\boldsymbol{r}_{j}$ in the coordinate system of the $j$ th bubble has coordinates $\left(r_{j}, \theta_{j}, \varepsilon_{j}\right)$. The position of the center of the $j$ th bubble with respect to the center of the cavity is denoted as $z_{j}$. In view of symmetry, the translational motion of the bubbles can only occur along the $z$ axis. The liquid in the cavity is assumed incompressible and inviscid so its motion is described by a scalar velocity potential that obeys the Laplace equation $\Delta \varphi=0$.

The mathematical derivation consists of the following main stages. We first find solutions for the liquid motion inside the cavity. Doing so, we use transformations between the coordinate systems described above in order to satisfy boundary conditions at the bubble surfaces and at the cavity surface. We then find solutions for the motion of the solid medium, assuming that the cavity shape keeps spherical at all times. As a result, four quantities remain unknown: the time-varying bubble radii and the positions of the bubble centers. To derive equations for these quantities, we apply the method of the Lagrangian formalism.

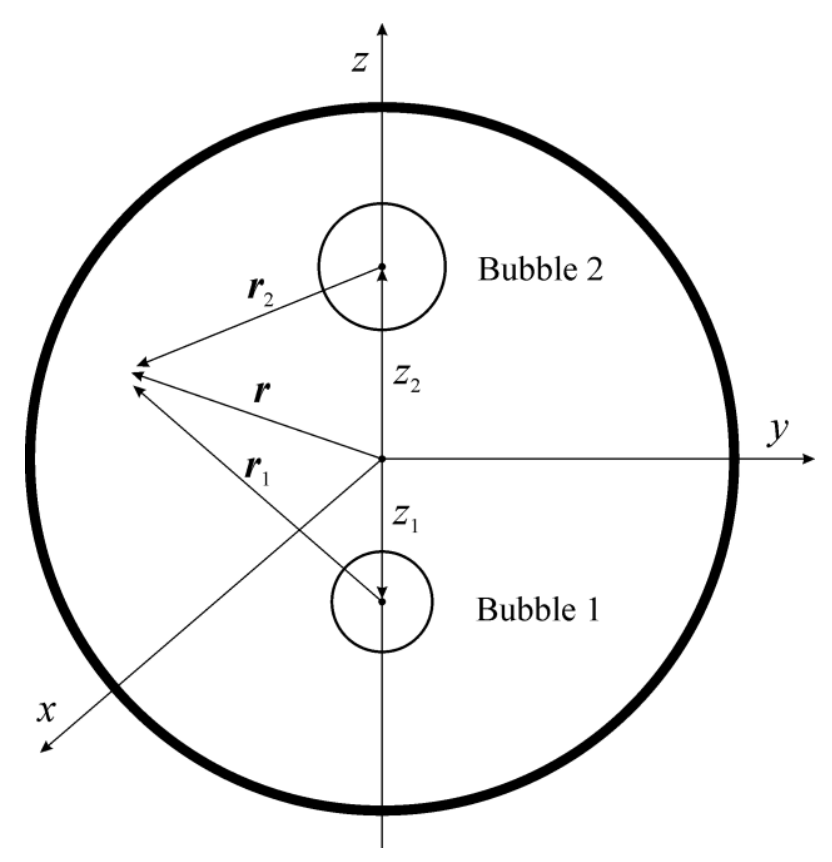

FIG. 1. Two bubbles inside a liquid-filled cavity.

\section{A. Solutions inside the cavity}

The total velocity potential in the liquid can be written as 


$$
\varphi=\varphi_{1}+\varphi_{2}+\varphi_{c}
$$

where $\varphi_{j}$ is the velocity potential of the $j$ th bubble and $\varphi_{c}$ is the velocity potential produced by the motion of the cavity surface. The expressions for $\varphi_{j}$ and $\varphi_{c}$, satisfying the Laplace equation, are given by

$$
\begin{gathered}
\varphi_{j}=\sum_{n=0}^{\infty} a_{n}^{(j)}(t)\left(\frac{R_{j}}{r_{j}}\right)^{n+1} P_{n}\left(\cos \theta_{j}\right), \quad j=1,2, \\
\varphi_{c}=\sum_{n=1}^{\infty} A_{n}(t)\left(\frac{r}{R_{c 0}}\right)^{n} P_{n}(\cos \theta),
\end{gathered}
$$

where $R_{j}(t)$ is the instantaneous radius of the $j$ th bubble, $R_{c 0}$ is the radius of the cavity at rest, and $P_{n}$ is the Legendre polynomial of order $n$.

The unknown functions $a_{n}^{(j)}$ and $A_{n}$ are calculated in Appendix A. To this end, boundary conditions at the bubble surfaces and at the cavity surface are used. As a result, recurrence formulas are derived that allow one to evaluate $a_{n}^{(j)}$ and $A_{n}$ with any desired accuracy; see Eqs. (A21) (A23) in Appendix A.

\section{B. Solutions in the solid}

The motion of the solid is described by the Navier equation [27],

$$
\rho_{s} \frac{\partial^{2} \boldsymbol{u}}{\partial t^{2}}=\mu \Delta \boldsymbol{u}+(\lambda+\mu) \nabla(\nabla \cdot \boldsymbol{u})
$$

where $\boldsymbol{u}$ is the displacement vector, $\partial \boldsymbol{u} / \partial t$ is the velocity in the solid, $\rho_{s}$ is the density of the solid, and $\lambda$ and $\mu$ are the Lamé coefficients. In view of the spherical shape of the cavity, $\boldsymbol{u}$ has only a radial component, $u(r, t)$, which can be written in terms of a potential $\varphi_{s}$ as

$$
u(r, t)=\frac{\partial \varphi_{s}}{\partial r}
$$

An expression for $\varphi_{s}$, satisfying Eq. (4), is written as

$$
\varphi_{s}=-\frac{s_{1}\left(t-r / c_{s}\right)}{r}-\frac{s_{2}\left(t+r / c_{s}\right)}{r}
$$

where $c_{s}=\sqrt{(\lambda+2 \mu) / \rho_{s}}$ is the longitudinal wave speed [27]. 
Substitution of Eq. (6) into Eq. (5) yields

$$
u(r, t)=\frac{s_{1}\left(t-r / c_{s}\right)+s_{2}\left(t+r / c_{s}\right)}{r^{2}}+\frac{s_{1}^{\prime}\left(t-r / c_{s}\right)-s_{2}^{\prime}\left(t+r / c_{s}\right)}{c_{s} r} .
$$

It is shown in Appendix B that, with an accuracy up to $1 / c_{s}^{2}$, Eq. (7) gives

$$
u(r, t)=\frac{R_{c 0}^{2}\left(R_{c}-R_{c 0}\right)}{r^{2}}+\frac{R_{c 0}^{2} \ddot{R}_{c}}{2 c_{s}^{2}}\left(\frac{R_{c 0}^{2}}{r^{2}}-1\right)
$$

where $R_{c}(t)$ is the instantaneous radius of the cavity. From Eq. (8), it follows that the velocity in the solid is calculated by

$$
\frac{\partial u(r, t)}{\partial t}=\frac{R_{c 0}^{2} \dot{R}_{c}}{r^{2}}+\frac{R_{c 0}^{2} \dddot{R}_{c}}{2 c_{s}^{2}}\left(\frac{R_{c 0}^{2}}{r^{2}}-1\right)
$$

\section{Equations for $\boldsymbol{R}_{j}$ and $z_{j}$}

The solutions obtained above are used to derive equations for the bubble radii $R_{j}(t)$ and the positions of the bubble centers $z_{j}(t)$. To this end, the Lagrangian formalism is applied. The derivation is performed in Appendix C. As a result, we are led to the following equations:

$$
\begin{gathered}
R_{1} \ddot{R}_{1}\left[1+F\left(R_{1}, z_{1}\right)\right]+\dot{R}_{1}^{2}\left[\frac{3}{2}+2 F\left(R_{1}, z_{1}\right)\right]-\frac{\dot{z}_{1}^{2}}{4}\left(1+\frac{2 R_{1}^{3}}{R_{c 0}^{3}}\right)-\frac{P_{1}}{\rho_{l}}+\frac{4 \mu}{\rho_{l}}\left(\frac{R_{c}}{R_{c 0}}-1\right)\left(1-\frac{R_{c 0}^{3}}{R_{s}^{3}}\right)-\frac{H}{c_{s}^{2}} \\
+\left(R_{2} \ddot{R}_{2}+2 \dot{R}_{2}^{2}\right) G\left(R_{2}, z_{1}, z_{2}\right)+\frac{R_{1}^{3} z_{1} \ddot{z}_{1}}{R_{c 0}^{3}}-R_{2} \ddot{z}_{2}\left(\frac{R_{2}^{2}}{2 d^{2}}-\frac{R_{2}^{2} z_{1}}{R_{c 0}^{3}}\right) \\
-\frac{R_{2}^{2} \dot{R}_{2}\left(\dot{z}_{1}+5 \dot{z}_{2}\right)}{2 d^{2}}+\frac{R_{2}^{3} \dot{z}_{2}\left(\dot{z}_{1}+2 \dot{z}_{2}\right)}{2 d^{3}}+\frac{8 R_{1}^{2} \dot{R}_{1} z_{1} \dot{z}_{1}+2 R_{2}^{2} \dot{R}_{2}\left(5 z_{1} \dot{z}_{2}-\dot{z}_{1} z_{2}\right)-R_{2}^{3} \dot{z}_{1} \dot{z}_{2}}{2 R_{c 0}^{3}}=0 \\
R_{2} \ddot{R}_{2}\left[1+F\left(R_{2}, z_{2}\right)\right]+\dot{R}_{2}^{2}\left[\frac{3}{2}+2 F\left(R_{2}, z_{2}\right)\right]-\frac{\dot{z}_{2}^{2}}{4}\left(1+\frac{2 R_{2}^{3}}{R_{c 0}^{3}}\right)-\frac{P_{2}}{\rho_{l}}+\frac{4 \mu}{\rho_{l}}\left(\frac{R_{c}}{R_{c 0}}-1\right)\left(1-\frac{R_{c 0}^{3}}{R_{s}^{3}}\right)-\frac{H}{c_{s}^{2}} \\
+\left(R_{1} \ddot{R}_{1}+2 \dot{R}_{1}^{2}\right) G\left(R_{1}, z_{1}, z_{2}\right)+\frac{R_{2}^{3} z_{2} \ddot{z}_{2}}{R_{c 0}^{3}}+R_{1} \ddot{z}_{1}\left(\frac{R_{1}^{2}}{2 d^{2}}+\frac{R_{1}^{2} z_{2}}{R_{c 0}^{3}}\right) \\
+\frac{R_{1}^{2} \dot{R}_{1}\left(\dot{z}_{2}+5 \dot{z}_{1}\right)}{2 d^{2}}+\frac{R_{1}^{3} \dot{z}_{1}\left(\dot{z}_{2}+2 \dot{z}_{1}\right)}{2 d^{3}}+\frac{8 R_{2}^{2} \dot{R}_{2} z_{2} \dot{z}_{2}+2 R_{1}^{2} \dot{R}_{1}\left(5 \dot{z}_{1} z_{2}-z_{1} \dot{z}_{2}\right)-R_{1}^{3} \dot{z}_{1} \dot{z}_{2}}{2 R_{c 0}^{3}}=0
\end{gathered}
$$




$$
\begin{aligned}
& \frac{R_{1} \ddot{z}_{1}}{3}\left(1+\frac{3 R_{1}^{3}}{R_{c 0}^{3}}\right)+\dot{R}_{1} \dot{z}_{1}\left(1+\frac{6 R_{1}^{3}}{R_{c 0}^{3}}\right)+\frac{2 R_{1}^{2} z_{1}\left(R_{1} \ddot{R}_{1}+3 \dot{R}_{1}^{2}\right)}{R_{c 0}^{3}} \\
& +R_{2}\left[R_{1}\left(R_{2} \ddot{R}_{2}+2 \dot{R}_{2}^{2}\right)+R_{2} \dot{R}_{1} \dot{R}_{2}\right]\left(\frac{2 z_{2}}{R_{c 0}^{3}}+\frac{1}{d^{2}}\right) \\
& +R_{2}^{2}\left[R_{1} R_{2} \ddot{z}_{2}+\dot{z}_{2}\left(R_{2} \dot{R}_{1}+5 R_{1} \dot{R}_{2}\right)\right]\left(\frac{1}{R_{c 0}^{3}}-\frac{1}{d^{3}}\right)=\frac{F_{D 1}}{2 \pi \rho_{l} R_{1}^{2}}, \\
& \frac{R_{2} \ddot{z}_{2}}{3}\left(1+\frac{3 R_{2}^{3}}{R_{c 0}^{3}}\right)+\dot{R}_{2} \dot{z}_{2}\left(1+\frac{6 R_{2}^{3}}{R_{c 0}^{3}}\right)+\frac{2 R_{2}^{2} z_{2}\left(R_{2} \ddot{R}_{2}+3 \dot{R}_{2}^{2}\right)}{R_{c 0}^{3}} \\
& \quad R_{1}\left[R_{2}\left(R_{1} \ddot{R}_{1}+2 \dot{R}_{1}^{2}\right)+R_{1} \dot{R}_{1} \dot{R}_{2}\right]\left(\frac{2 z_{1}}{R_{c 0}^{3}}-\frac{1}{d^{2}}\right) \\
& +R_{1}^{2}\left[R_{1} R_{2} \ddot{z}_{1}+\dot{z}_{1}\left(R_{1} \dot{R}_{2}+5 R_{2} \dot{R}_{1}\right)\right]\left(\frac{1}{R_{c 0}^{3}}-\frac{1}{d^{3}}\right)=\frac{F_{D 2}}{2 \pi \rho_{l} R_{2}^{2}},
\end{aligned}
$$

where $\rho_{l}$ is the liquid density, $\mu$ is the shear modulus of the solid, $d(t)=z_{2}(t)-z_{1}(t)$ is the distance between the bubble centers, $P_{j}$ is the scattered pressure at the surface of the $j$ th bubble, which is given by Eq. (C5), $F_{D j}$ is the viscous drag force on the $j$ th bubble, which is given by Eq. (C6), and the functions $F, G$, and $H$ are defined by Eqs. (C33), (C34), and (C44), respectively.

Equations (10) and (11) govern the coupled pulsations of the bubbles and Eqs. (12) and (13) govern their translational motion. All these equations are ordinary differential equations of second order with respect to time derivatives. They form a combined system and should be solved simultaneously.

\section{Linearized equations}

The aim of this subsection is to simplify Eqs. (10) and (11) in order to make them amenable to analytical consideration. To this end, we linearize them, neglecting the translational motion and the compressibility corrections in the liquid and in the solid. We also assume that $R_{s} \gg R_{c 0}$, where $R_{s}$ denotes the external radius of the solid layer (see Appendix C).

We set

$$
R_{j}=R_{j 0}+x_{j}(t), \quad R_{c}=R_{c 0}+x_{c}(t)
$$


where $R_{j 0}$ denotes the equilibrium radius of the $j$ th bubble, and we assume that $\left|x_{j}\right|<<R_{j 0}$ and $\left|x_{c}\right|<<R_{c 0}$.

Substituting Eq. (14) into Eqs. (10) and (11), using the results of Appendix C, and keeping up to terms of first order in $x_{j}$ and $x_{c}$, one obtains

$$
\begin{aligned}
& \ddot{x}_{1}+\delta_{1} \dot{x}_{1}+\omega_{10}^{2} x_{1}+\frac{R_{20}}{R_{10} \alpha_{1}}\left(\beta_{2} \ddot{x}_{2}+\alpha_{2} \Omega_{2}^{2} x_{2}\right)=-\frac{P_{a c}}{\rho_{l} R_{10} \alpha_{1}}, \\
& \ddot{x}_{2}+\delta_{2} \dot{x}_{2}+\omega_{20}^{2} x_{2}+\frac{R_{10}}{R_{20} \alpha_{2}}\left(\beta_{1} \ddot{x}_{1}+\alpha_{1} \Omega_{1}^{2} x_{1}\right)=-\frac{P_{a c}}{\rho_{l} R_{20} \alpha_{2}},
\end{aligned}
$$

where

$$
\begin{gathered}
\omega_{j 0}^{2}=\frac{1}{\rho_{l} R_{j 0}^{2} \alpha_{j}}\left(3 \gamma P_{g j}-\frac{2 \sigma_{l}}{R_{j 0}}\right)+\Omega_{j}^{2}, \\
\Omega_{j}^{2}=\frac{4 \mu R_{j 0}}{\rho_{l} R_{c 0}^{3} \alpha_{j}}, \\
\delta_{j}=\frac{4 \eta_{l}}{\rho_{l} R_{j 0}^{2} \alpha_{j}}, \\
\alpha_{j}=1+\frac{R_{j 0}}{R_{c 0}}\left(1+\frac{\rho_{s}}{\rho_{l}}\right)+\frac{2 R_{j 0} z_{j 0}^{2}}{R_{c 0}^{3}}, \\
\beta_{j}=\frac{R_{j 0}}{d}+\frac{R_{j 0}}{R_{c 0}}\left(1+\frac{\rho_{s}}{\rho_{l}}\right)+\frac{2 R_{j 0} z_{10} z_{20}}{R_{c 0}^{3}} .
\end{gathered}
$$

Definitions of all the quantities that appear in these equations are given in Appendix C. Note that for $R_{c 0} \rightarrow \infty$, the first term in Eq. (17) gives the natural frequency of the $j$ th bubble in an unbounded liquid.

In order to calculate the eigenfrequencies of the system of Eqs. (15) and (16), we set the external acoustic excitation equal to zero $\left(P_{a c}=0\right)$ and $x_{j}=a_{j} \exp (-i \omega t)$. As a result, Eqs. (15) and (16) reduce to

$$
\begin{aligned}
& \left(\omega^{2}-\omega_{10}^{2}+i \omega \delta_{1}\right) a_{1}+\frac{R_{20}}{R_{10} \alpha_{1}}\left(\beta_{2} \omega^{2}-\alpha_{2} \Omega_{2}^{2}\right) a_{2}=0 \\
& \frac{R_{10}}{R_{20} \alpha_{2}}\left(\beta_{1} \omega^{2}-\alpha_{1} \Omega_{1}^{2}\right) a_{1}+\left(\omega^{2}-\omega_{20}^{2}+i \omega \delta_{2}\right) a_{2}=0 .
\end{aligned}
$$


An equation for calculating the eigenfrequencies is derived setting the determinant of Eqs. (22) and (23) equal to zero. This operation results in

$$
\left(\omega^{2}-\omega_{10}^{2}+i \omega \delta_{1}\right)\left(\omega^{2}-\omega_{20}^{2}+i \omega \delta_{2}\right)-\frac{1}{\alpha_{1} \alpha_{2}}\left(\beta_{1} \omega^{2}-\alpha_{1} \Omega_{1}^{2}\right)\left(\beta_{2} \omega^{2}-\alpha_{2} \Omega_{2}^{2}\right)=0 .
$$

As one can see, Eq. (24) is an equation of the forth order in $\omega$. In the general case, a numerical solution of this equation is only possible. Yet, for two identical bubbles, $R_{10}=R_{20}$, an analytical solution can be obtained. In this case, neglecting the viscous dissipation $\left(\delta_{j}=0\right)$, Eq. (24) reduces to

$$
\left(1-\frac{\beta_{1}^{2}}{\alpha_{1}^{2}}\right) \omega^{4}-\frac{2}{\alpha_{1}}\left(\alpha_{1} \omega_{10}^{2}-\beta_{1} \Omega_{1}^{2}\right) \omega^{2}+\omega_{10}^{4}-\Omega_{1}^{4}=0 .
$$

The roots of this equation are given by

$$
\omega_{1,2}^{2}=\frac{\alpha_{1} \omega_{10}^{2}-\beta_{1} \Omega_{1}^{2} \pm\left|\alpha_{1} \Omega_{1}^{2}-\beta_{1} \omega_{10}^{2}\right|}{\alpha_{1}\left(1-\beta_{1}^{2} / \alpha_{1}^{2}\right)} .
$$

If $R_{10}, z_{j 0}<<R_{c 0}$ and $R_{10}<<d$, Eq. (26) is simplified to

$$
\omega_{1,2}^{2}=\omega_{10}^{2}-\beta_{1} \Omega_{1}^{2} \pm\left|\Omega_{1}^{2}-\beta_{1} \omega_{10}^{2}\right| .
$$

If $\beta_{1}$, given by Eq. (21), is sufficiently small, one can write

$$
\omega_{1}^{2} \approx \omega_{10}^{2}+\Omega_{1}^{2}, \quad \omega_{2}^{2} \approx \omega_{10}^{2}-\Omega_{1}^{2} .
$$

From Eq. (17) it follows that

$$
\omega_{1}^{2} \approx \omega_{M}^{2}+2 \Omega_{1}^{2}, \quad \omega_{2} \approx \omega_{M},
$$

where

$$
\omega_{M}=\frac{1}{R_{10}} \sqrt{\frac{3 \gamma P_{g 1}}{\rho_{l}}-\frac{2 \sigma_{l}}{\rho_{l} R_{10}}} .
$$

Equation (30) is the natural frequency of a single bubble in an unbounded liquid. This frequency is known as the Minnaert frequency, although, strictly speaking, the original Minnaert formula ignores surface tension [28].

Analysis reveals that the bubbles pulsate with the frequency $\omega_{1}$ in phase and with the frequency $\omega_{2}$ in antiphase. In many cases of interest, $\omega_{M}<<\Omega_{1}$. In such cases, the natural frequency of a single bubble in a cavity is approximately equal to $\Omega_{1}$ given by Eq. $(18)[13,15]$. 
This means that the in-phase free oscillation of two bubbles in a cavity is $\sqrt{2}$ times faster than that of a single bubble. The antiphase free oscillation of two bubbles in a cavity occurs at the Minnaert frequency.

In the limit $R_{c 0} \rightarrow \infty$, Eq. (27) gives

$$
\omega_{1,2}=\omega_{M}\left(1 \pm \frac{R_{10}}{2 d}\right) .
$$

This is the well-known equation for the eigenfrequencies of two identical bubbles separated by distance $d$ in an unbounded liquid [29].

\section{NUMERICAL SIMULATIONS}

Numerical simulations have been carried out by means of the program package MATHEMATICA (Wolfram Research, Champaign, IL). The following values of the physical parameters were used: $\rho_{l}=1000 \mathrm{~kg} / \mathrm{m}^{3}, c_{l}=1500 \mathrm{~m} / \mathrm{s}, \eta_{l}=0.001 \mathrm{~Pa} \mathrm{~s}, \sigma_{l}=0.072 \mathrm{~N} / \mathrm{m}$, $\rho_{s}=800 \mathrm{~kg} / \mathrm{m}^{3}, c_{s}=4000 \mathrm{~m} / \mathrm{s}, \mu=0.75 \mathrm{GPa}, P_{0}=101.3 \mathrm{kPa}$, and $\gamma=1.4$. The parameters of the liquid correspond to water and the parameters of the solid correspond to wood (oak). We have chosen wood as a surrounding medium because one of the most interesting applications where one has to deal with bubbles in a cavity is the investigation of cavitation events inside tree trunks $[6,7,9,10]$.

The main subject of interest in our numerical simulations is the acoustic response (resonance frequencies) of a two-bubble system in a cavity in comparison with the same case in an unbounded liquid. Because it is the acoustic response that is of prime interest in most applications.

Excitation is produced by an acoustic pressure pulse shown in Fig. 2. That is a Gaussian pulse given by

$$
P_{a c}(t)=P_{a} \sin (2 \pi f t) e^{-(2 f t / N)^{4}},
$$

where $P_{a}$ is the amplitude, $f$ is the frequency, and $N$ is the number of cycles. In Fig. $2, P_{a}=50$ $\mathrm{kPa}, f=1.5 \mathrm{MHz}$, and $N=5$.

Figures 3(a) - 3(c) show the response of two bubbles confined in a cavity to the acoustic pulse depicted in Fig. 2. It is assumed that $R_{10}=7.5 \mu \mathrm{m}, R_{20}=5 \mu \mathrm{m}, z_{1}(0)=-50 \mu \mathrm{m}, z_{2}(0)=50$ 
$\mu \mathrm{m}, R_{c 0}=100 \mu \mathrm{m}$, and $R_{s}=500 \mu \mathrm{m}$. For comparison, Figs. 3(d) $-3(\mathrm{f})$ show the response of the same bubbles in an unbounded liquid. Figures 3(a) and 3(b) show the radial oscillation of bubbles 1 and 2, respectively. Figure 3(c) shows the spectra of the oscillation curves. Comparison with the curves presented in Figs. 3(d) - 3(f) reveals considerable differences from the behavior of the bubbles in an unbounded liquid. As one can see, in an unbounded liquid, the forced oscillation in response to the imposed pulse ceases almost right after the termination of the pulse and the bubbles begin to execute free oscillations with proper natural frequencies. This is confirmed by two sharp spectral peaks at the left in Fig. 3(f). The Minnaert frequencies of the bubbles, i.e., the linear natural frequencies of the bubbles when they are far apart in an unbounded liquid, are $468 \mathrm{kHz}$ for bubble 1 and $724 \mathrm{kHz}$ for bubble 2. In the cavity, as is seen in Fig. 3(c), the forced oscillation turns into a free oscillation with a frequency of about $1 \mathrm{MHz}$, which is experienced by both bubbles. Calculations show that this oscillation gradually disappears with increasing $R_{c 0}$.

Figure 4 illustrates the translational motion of the bubbles, the parameters being the same as in Fig. 3, except that the number of cycles in the excitation pulse was set equal to $N=500$ in order to increase the translational shift. The solid line shows the motion of the bubbles in the cavity, whereas the dashed line shows their motion in an unbounded liquid. The bubbles are moving toward each other. As one can see, the approach of the bubbles in the cavity occurs faster.

As said above, the Minnaert frequencies of the bubbles shown in Figs. 3 and 4 are $468 \mathrm{kHz}$ for bubble 1 and $724 \mathrm{kHz}$ for bubble 2. This means that the bubbles in Figs. 3 and 4 are excited above resonance. Figures 5 and 6 illustrate the case that the driving frequency, $f=600 \mathrm{kHz}$, is in between the natural frequencies of the bubbles, the other parameters being the same as in Figs. 3 and 4, except that $N=50$ in Fig. 6 . We again observe a considerable difference between the pulsations of the bubbles in the cavity and in an unbounded liquid. The difference is especially visible when we compare the spectra shown in Figs. 5(c) and 5(f). It is interesting to note that in the cavity, the natural frequencies of the bubbles do not manifest themselves even after the acoustic pulse is over. Figure 6 shows the translational motion of the bubbles. As one can see, the bubbles are moving away from each other, the terminal separation between the bubbles in the cavity being greater than that in an unbounded liquid.

Figures 7 and 8 show the behavior of the bubbles at $f=300 \mathrm{kHz}$, the other parameters being the same as in Figs. 3 and 4, except that $N=1000$ in Fig. 8. In this case, the bubbles are 
excited below their natural frequencies. Figure 7(f) predicts strong second and third harmonics in an unbounded liquid. In the cavity, according to Fig. 7(c), the second harmonic only develops. Note also that the oscillation amplitude in the cavity is much smaller than that in an unbounded liquid. The translational motion, presented in Fig. 8, demonstrates that in an unbounded liquid, the acoustic pulse makes the bubbles approach, whereas in the cavity, the bubbles in fact stay where they are, showing a weak tendency to move away from each other.

Figures 9 and 10 exemplify free oscillations, which are excited at $P_{a}=0$ by a small initial deviation from the equilibrium bubble radii. Figure 9 demonstrates the case of equal bubbles with $R_{10}=R_{20}=5 \mu \mathrm{m}$. The Minnaert frequency corresponding to this size is $724 \mathrm{kHz}$. Other parameters are $z_{1}(0)=-50 \mu \mathrm{m}, z_{2}(0)=50 \mu \mathrm{m}, R_{c 0}=100 \mu \mathrm{m}$, and $R_{s}=500 \mu \mathrm{m}$. Figure 9(d) shows that in an unbounded liquid, the bubbles pulsate at a frequency of $706 \mathrm{kHz}$. As would be expected, this value corresponds to Eq. (31). In the cavity, as follows from Fig. 9(b), the bubbles pulsate at 1.025 MHz. This value is well approximated by Eq. (26). Note that in the case under study, $\omega_{M}$ is not small compared to $\Omega_{1}$, so the approximate expressions derived in the last but one paragraph of Sec. II D are not valid.

Figure 10 illustrates the case of unequal bubbles with $R_{10}=7.5 \mu \mathrm{m}$ and $R_{20}=5 \mu \mathrm{m}$, the other parameters being the same as in Fig. 9. As one can see, in an unbounded liquid, the bubbles pulsate at frequencies close to their Minnaert frequencies, whereas in the cavity, they pulsate at different frequencies. This result confirms the analytical predictions made in Sec. II D that the confinement in a cavity fundamentally changes the eigenfrequencies of a two-bubble system.

\section{EXPERIMENTS IN CLOSED RECTANGULAR CHANNELS}

In this section, we present experiments for two confined bubbles freely oscillating after nucleation. The geometry of the confinement is different from that considered in our theory. However, we suppose that the main physical factor in the problem under study is the presence of full confinement as such rather than its concrete form. In other words, in the experiments just as in our theory, bubbles are coupled by a finite liquid volume enclosed in an elastic medium. We believe that the qualitative similarity of physical conditions should give rise to a similarity in the qualitative behavior of the bubbles. 
The microchannels are fabricated in pHEMA hydrogel using the method by Wheeler and Stroock [30]. The channels are filled with water after soaking in degassed water over one night. The channels are then placed in a dry atmosphere for 12 hours with a humidity level imposed by the method of salt solutions. Under drying, the hydrogel becomes extremely stiff, resisting an intense negative pressure (about - $5 \mathrm{MPa}$ ) that progressively builds up in the liquid contained in the channel when it per-evaporates through the channel. Bubble nucleation is triggered by a laser pulse shot in the hydrogel near the channel, using the same set-up as that described in works of Vincent et al. [11] and Vincent and Marmottant [13]. A high-speed camera, synchronized with the laser pulse, records four images before the bubble nucleation and then the growth of the bubbles, see Fig. 11.

The data acquired in the above experiments can be compared to our theory. Note that in the experiments, the hydrostatic liquid pressure was not constant. It was initially negative and then it was relaxing following the bubble growth and the stress relaxation in the solid. Based on the results of Vincent and Marmottant [13], the relaxation of the hydrostatic liquid pressure can be approximated by the following expression:

$$
P_{l}(t)=P_{0}+K\left(\frac{R_{1}}{R_{c 0}}\right)^{3}+K\left(\frac{R_{2}}{R_{c 0}}\right)^{3},
$$

where $K$ is an effective solid modulus.

In our theory, the driving pressure is set by $P_{0}+P_{a c}(t)$; see Eq. (C5). In order to model the relaxation of the hydrostatic pressure, we replace $P_{0}+P_{a c}(t)$ with Eq. (33). Based on the experimental measurements, the physical parameters used in our simulation were set as follows: $\rho_{l}=1000 \mathrm{~kg} / \mathrm{m}^{3}, c_{l}=1500 \mathrm{~m} / \mathrm{s}, \eta_{l}=0.01 \mathrm{~Pa} \mathrm{~s}, \sigma_{l}=0.027 \mathrm{~N} / \mathrm{m}, \rho_{s}=1233 \mathrm{~kg} / \mathrm{m}^{3}, c_{s}=2111$ $\mathrm{m} / \mathrm{s}, \gamma=1, P_{0}=-10 \mathrm{kPa}, K=8 \mathrm{MPa}, \mu=(3 / 4) K, R_{c 0}=450 \mu \mathrm{m}, R_{s}=10^{3} \mu \mathrm{m}, z_{1}(0)=-225$ $\mu \mathrm{m}, z_{2}(0)=225 \mu \mathrm{m}, R_{10}=6 \mu \mathrm{m}, R_{20}=5.95 \mu \mathrm{m}$, and $P_{g j}=p_{g 0}+2 \sigma_{l} / R_{j 0}$ with $p_{g 0}=1 \mathrm{kPa}$. The values of $P_{0}, K, R_{10}$, and $R_{20}$, which could not be exactly measured, were used as fitting parameters.

The comparison of the experimental results, shown by circles and squares, with theoretical predictions, shown by solid and dashed curves, is presented in Fig. 12. In a cavity, regardless of its shape, there is a strong coupling between the volumes of bubbles because the sum of the bubble volumes must remain constant if the compressibility of the liquid is negligible. This effect is 
demonstrated by Fig. 12. We see that, if the equilibrium volume of one bubble increases, that of the other bubble decreases. Such a coupling is absent for bubbles in an unbounded liquid and therefore the acoustic interaction of bubbles in an unbounded liquid does not change their equilibrium radii. As Fig. 12 shows, in spite of the different form of the confinement, the behavior of the bubbles in the experiment demonstrates the same qualitative features as those predicted by the theory for a spherical confinement.

\section{CONCLUSIONS}

In the present study, a theory has been developed that allows one to model the nonlinear dynamics of two coupled gas bubbles inside a spherical liquid-filled cavity surrounded by an elastic medium. The aim of the study was to reveal how the conditions of full confinement affected the coupled oscillations of the bubbles. To make the problem amenable to analytical consideration, the bubbles were assumed to be located on a diameter of the cavity. This assumption makes the problem axisymmetric, which simplifies calculations. Combined equations for the pulsation and translation motion of the bubbles were derived. The derivation was carried out by the Lagrangian formalism. The derived equations were then used in numerical simulations. The behavior of two bubbles in a cavity was compared with the behavior of the same bubbles in an unbounded liquid. The comparison has revealed that both forced and free oscillations of two bubbles in a cavity occur differently than those in an unbounded liquid. In particular, the eigenfrequencies of a two-bubble system in a cavity are found to be different from those in an unbounded liquid.

\section{ACKNOWLEDGMENT}

This research has received funding from the European Research Council under the European Union's Seventh Framework Programme (FP7/2007-2013)/ERC Grant Agreement No. 614655 "Bubbleboost".

\section{APPENDIX A: CALCULATION OF $a_{n}^{(j)}(t)$ AND $A_{n}(t)$}

In this Appendix, we calculate the functions $a_{n}^{(j)}(t)$ and $A_{n}(t)$ that appear in Eqs. (2) and (3). To this end, we apply boundary conditions at the bubble surfaces and at the cavity surface. 
To satisfy the boundary conditions at the bubble surfaces, we need an expression for $\varphi_{1}$ in terms of the coordinates of bubble 2 and vice versa. These expressions can be written as

$$
\begin{aligned}
& \varphi_{1}=\sum_{n=0}^{\infty} b_{n}^{(1)}(t)\left(\frac{r_{2}}{d}\right)^{n} P_{n}\left(\cos \theta_{2}\right), \\
& \varphi_{2}=\sum_{n=0}^{\infty} b_{n}^{(2)}(t)\left(\frac{r_{1}}{d}\right)^{n} P_{n}\left(\cos \theta_{1}\right),
\end{aligned}
$$

where $b_{n}^{(j)}$ is a time function to be determined and $d(t)=z_{2}(t)-z_{1}(t)$ is the distance between the bubble centers. Note that $z_{2}$ is taken to be greater than $z_{1}$ in order to keep $d$ positive.

To find $b_{n}^{(j)}$, we apply the following mathematical identities [31]:

$$
\begin{aligned}
& \frac{P_{n}\left(\cos \theta_{1}\right)}{r_{1}^{n+1}}=\sum_{m=0}^{\infty} \frac{(-1)^{m}(n+m) !}{d^{n+1} n ! m !}\left(\frac{r_{2}}{d}\right)^{m} P_{m}\left(\cos \theta_{2}\right), \\
& \frac{P_{n}\left(\cos \theta_{2}\right)}{r_{2}^{n+1}}=\sum_{m=0}^{\infty} \frac{(-1)^{n}(n+m) !}{d^{n+1} n ! m !}\left(\frac{r_{1}}{d}\right)^{m} P_{m}\left(\cos \theta_{1}\right) .
\end{aligned}
$$

Substituting Eq. (A3) into Eq. (2) at $j=1$ and comparing with Eq. (A1), one obtains

$$
b_{n}^{(1)}=\sum_{m=0}^{\infty} \frac{(-1)^{n}(n+m) !}{n ! m !} \xi_{1}^{m+1} a_{m}^{(1)},
$$

where $\xi_{1}=R_{1} / d$.

Substituting Eq. (A4) into Eq. (2) at $j=2$ and comparing with Eq. (A2), one obtains

$$
b_{n}^{(2)}=\sum_{m=0}^{\infty} \frac{(-1)^{m}(n+m) !}{n ! m !} \xi_{2}^{m+1} a_{m}^{(2)},
$$

where $\xi_{2}=R_{2} / d$.

To satisfy the boundary condition at the cavity surface, we need an expression for $\varphi_{j}$ in terms of the global coordinates. It can be written as

$$
\varphi_{j}=\sum_{n=0}^{\infty} c_{n}^{(j)}(t)\left(\frac{R_{c 0}}{r}\right)^{n+1} P_{n}(\cos \theta) .
$$

To find $c_{n}^{(j)}$, we use the following identity [31]:

$$
\frac{P_{n}\left(\cos \theta_{j}\right)}{r_{j}^{n+1}}=\sum_{m=n}^{\infty} \frac{m ! z_{j}^{m-n}}{n !(m-n) !} \frac{P_{m}(\cos \theta)}{r^{m+1}} .
$$


Substituting Eq. (A8) into Eq. (2) and comparing with Eq. (A7), one obtains

$$
c_{n}^{(j)}=\sum_{m=0}^{n} \frac{n ! z_{j}^{n-m} R_{j}^{m+1}}{m !(n-m) ! R_{c 0}^{n+1}} a_{m}^{(j)} .
$$

We also need an expression for $\varphi_{c}$ in terms of the coordinates of the $j$ th bubble. This expression is given by

$$
\varphi_{c}=\sum_{n=0}^{\infty} A_{n}^{(j)}(t)\left(\frac{r_{j}}{R_{j}}\right)^{n} P_{n}\left(\cos \theta_{j}\right) .
$$

To find $A_{n}^{(j)}$, we use the following identity [31]:

$$
r^{n} P_{n}(\cos \theta)=\sum_{m=0}^{n} \frac{n ! z_{j}^{n-m}}{m !(n-m) !} r_{j}^{m} P_{m}\left(\cos \theta_{j}\right) .
$$

Substituting Eq. (A11) into Eq. (3) and comparing with Eq. (A10), one obtains

$$
A_{n}^{(j)}=\sum_{\substack{m=1 \\ m \geq n}}^{\infty} \frac{m ! z_{j}^{m-n} R_{j}^{n}}{n !(m-n) ! R_{c 0}^{m}} A_{m} .
$$

We can now apply the boundary conditions. The boundary condition for the normal velocity at the surface of the $j$ th bubble is given by

$$
\frac{\partial \varphi}{\partial r_{j}}=\dot{R}_{j}+\dot{z}_{j} \cos \theta_{j} \text { at } r_{j}=R_{j},
$$

where the overdot means the time derivative. On substitution of Eqs. (2), (A1), (A2), and (A10), Eq. (A13) gives

$$
\begin{aligned}
& a_{n}^{(1)}=-R_{1} \dot{R}_{1} \delta_{n 0}-\frac{1}{2} R_{1} \dot{z}_{1} \delta_{n 1}+\frac{n}{n+1}\left(\xi_{1}^{n} b_{n}^{(2)}+A_{n}^{(1)}\right), \\
& a_{n}^{(2)}=-R_{2} \dot{R}_{2} \delta_{n 0}-\frac{1}{2} R_{2} \dot{z}_{2} \delta_{n 1}+\frac{n}{n+1}\left(\xi_{2}^{n} b_{n}^{(1)}+A_{n}^{(2)}\right),
\end{aligned}
$$

where $\delta_{n m}$ is the Kronecker delta.

The boundary condition for the normal velocity at the inner cavity surface is given by

$$
\frac{\partial \varphi}{\partial r}=\dot{R}_{c} \text { at } r=R_{c 0},
$$

where $R_{c}(t)$ is the instantaneous radius of the cavity. As is the convention in the linear theory of elasticity, we assume that the deformation of the solid is small and therefore the boundary condition is applied to the cavity surface at rest. 
Substituting Eqs. (3) and (A7) into Eq. (A16), one finds

$$
c_{n}^{(1)}+c_{n}^{(2)}=-R_{c 0} \dot{R}_{c} \delta_{n 0}+\frac{n}{n+1} A_{n} .
$$

Substitution of Eq. (A9) into Eq. (A17) yields

$$
-R_{c 0} \dot{R}_{c} \delta_{n 0}+\frac{n}{n+1} A_{n}=\frac{n !}{R_{c 0}^{n+1}} \sum_{m=0}^{n} \frac{R_{1}^{m+1} z_{1}^{n-m} a_{m}^{(1)}+R_{2}^{m+1} z_{2}^{n-m} a_{m}^{(2)}}{m !(n-m) !} .
$$

From Eq. (A18) for $n=0$, using Eqs. (A14) and (A15), one obtains

$$
R_{c 0}^{2} \dot{R}_{c}=R_{1}^{2} \dot{R}_{1}+R_{2}^{2} \dot{R}_{2}
$$

It is worth noting that Eq. (A19) expresses the conservation of the liquid volume, which follows from the assumption of liquid incompressibility.

From Eq. (A18) for $n \geq 1$ follows

$$
A_{n}=\frac{(n+1)(n-1) !}{R_{c 0}^{n+1}} \sum_{m=0}^{n} \frac{R_{1}^{m+1} z_{1}^{n-m} a_{m}^{(1)}+R_{2}^{m+1} z_{2}^{n-m} a_{m}^{(2)}}{m !(n-m) !} \text { for } n \geq 1 .
$$

Substitution of Eq. (A20) into Eq. (A12) gives an expression for $A_{n}^{(j)}$ in terms of $a_{n}^{(j)}$,

$$
A_{n}^{(j)}=\frac{R_{j}^{n}}{n !} \sum_{\substack{m=1 \\ m \geq n}}^{\infty} \sum_{l=0}^{m} \frac{(m-1) !(m+1) !}{l !(m-n) !(m-l) !} \frac{z_{j}^{m-n}}{R_{c 0}^{2 m+1}}\left(R_{1}^{l+1} z_{1}^{m-l} a_{l}^{(1)}+R_{2}^{l+1} z_{2}^{m-l} a_{l}^{(2)}\right) .
$$

Substituting Eqs. (A6) and (A21) into Eq. (A14), one obtains

$$
\begin{gathered}
a_{n}^{(1)}=-R_{1} \dot{R}_{1} \delta_{n 0}-\frac{1}{2} R_{1} \dot{z}_{1} \delta_{n 1}+\frac{n \xi_{1}^{n}}{(n+1) !} \sum_{m=0}^{\infty} \frac{(-1)^{m}(n+m) !}{m !} \xi_{2}^{m+1} a_{m}^{(2)} \\
+\frac{n R_{1}^{n}}{(n+1) !} \sum_{\substack{m=1 \\
m \geq n}}^{\infty} \sum_{l=0}^{m} \frac{(m-1) !(m+1) !}{l !(m-n) !(m-l) !} \frac{z_{1}^{m-n}}{R_{c 0}^{2 m+1}}\left(R_{1}^{l+1} z_{1}^{m-l} a_{l}^{(1)}+R_{2}^{l+1} z_{2}^{m-l} a_{l}^{(2)}\right) .
\end{gathered}
$$

Substituting Eqs. (A5) and (A21) into Eq. (A15), one obtains

$$
\begin{gathered}
a_{n}^{(2)}=-R_{2} \dot{R}_{2} \delta_{n 0}-\frac{1}{2} R_{2} \dot{z}_{2} \delta_{n 1}+\frac{(-1)^{n} n \xi_{2}^{n}}{(n+1) !} \sum_{m=0}^{\infty} \frac{(n+m) !}{m !} \xi_{1}^{m+1} a_{m}^{(1)} \\
+\frac{n R_{2}^{n}}{(n+1) !} \sum_{\substack{m=1 \\
m \geq n}}^{\infty} \sum_{l=0}^{m} \frac{(m-1) !(m+1) !}{l !(m-n) !(m-l) !} \frac{z_{2}^{m-n}}{R_{c 0}^{2 m+1}}\left(R_{1}^{l+1} z_{1}^{m-l} a_{l}^{(1)}+R_{2}^{l+1} z_{2}^{m-l} a_{l}^{(2)}\right) .
\end{gathered}
$$

Equations (A22) and (A23) allow one to calculate $a_{n}^{(j)}$ with any desired accuracy, considering $\xi_{j}, R_{j} / R_{c 0}$, and $z_{j} / R_{c 0}$ as expansion parameters and using a recurrence procedure. 


\section{APPENDIX B: CALCULATION OF THE DISPLACEMENT IN THE SOLID}

We apply the boundary condition for the displacement at the cavity surface,

$$
u(r, t)=R_{c}(t)-R_{c 0} \text { at } r=R_{c 0},
$$

where $R_{c}(t)$ is the instantaneous radius of the cavity. Substitution of Eq. (7) into Eq. (B1) yields

$$
s_{1}\left(t-R_{c 0} / c_{s}\right)+s_{2}\left(t+R_{c 0} / c_{s}\right)+\frac{R_{c 0}}{c_{s}}\left[s_{1}^{\prime}\left(t-R_{c 0} / c_{s}\right)-s_{2}^{\prime}\left(t+R_{c 0} / c_{s}\right)\right]=R_{c 0}^{2}\left(R_{c}-R_{c 0}\right),
$$

where the prime denotes the derivative with respect to the argument in brackets. From Eq. (B2), it follows that

$$
s_{1}^{\prime}\left(t-R_{c 0} / c_{s}\right)+s_{2}^{\prime}\left(t+R_{c 0} / c_{s}\right)+\frac{R_{c 0}}{c_{s}}\left[s_{1}^{\prime \prime}\left(t-R_{c 0} / c_{s}\right)-s_{2}^{\prime \prime}\left(t+R_{c 0} / c_{s}\right)\right]=R_{c 0}^{2} \dot{R}_{c} .
$$

Equations (B2) and (B3) allow one to calculate $u(r, t)$ with an accuracy up to $1 / c_{s}^{2}$.

Let us expand $u(r, t)$ into a Taylor series at $r=R_{c 0}$ as follows:

$$
\begin{gathered}
u(r, t)=\frac{s_{1}\left[t-R_{c 0} / c_{s}-\left(r-R_{c 0}\right) / c_{s}\right]+s_{2}\left[t+R_{c 0} / c_{s}+\left(r-R_{c 0}\right) / c_{s}\right]}{r^{2}} \\
+\frac{s_{1}^{\prime}\left[t-R_{c 0} / c_{s}-\left(r-R_{c 0}\right) / c_{s}\right]-s_{2}^{\prime}\left[t+R_{c 0} / c_{s}+\left(r-R_{c 0}\right) / c_{s}\right]}{c_{s} r} \\
\approx \frac{1}{r^{2}}\left[s_{1}\left(t-R_{c 0} / c_{s}\right)+s_{2}\left(t+R_{c 0} / c_{s}\right)\right]-\frac{r-R_{c 0}}{c_{s} r^{2}}\left[s_{1}^{\prime}\left(t-R_{c 0} / c_{s}\right)-s_{2}^{\prime}\left(t+R_{c 0} / c_{s}\right)\right] \\
+\frac{\left(r-R_{c 0}\right)^{2}}{2 c_{s}^{2} r^{2}}\left[s_{1}^{\prime \prime}\left(t-R_{c 0} / c_{s}\right)+s_{2}^{\prime \prime}\left(t+R_{c 0} / c_{s}\right)\right] \\
+\frac{1}{c_{s} r}\left[s_{1}^{\prime}\left(t-R_{c 0} / c_{s}\right)-s_{2}^{\prime}\left(t+R_{c 0} / c_{s}\right)\right]-\frac{r-R_{c 0}}{c_{s}^{2} r}\left[s_{1}^{\prime \prime}\left(t-R_{c 0} / c_{s}\right)+s_{2}^{\prime \prime}\left(t+R_{c 0} / c_{s}\right)\right] .
\end{gathered}
$$

With the help of Eqs. (B2) and (B3), we finally obtain

$$
\begin{gathered}
u(r, t) \approx \frac{R_{c 0}^{2}\left(R_{c}-R_{c 0}\right)}{r^{2}}+\frac{1}{2 c_{s}^{2}}\left(\frac{R_{c 0}^{2}}{r^{2}}-1\right)\left[s_{1}^{\prime \prime}\left(t-R_{c 0} / c_{s}\right)+s_{2}^{\prime \prime}\left(t+R_{c 0} / c_{s}\right)\right] \\
\approx \frac{R_{c 0}^{2}\left(R_{c}-R_{c 0}\right)}{r^{2}}+\frac{R_{c 0}^{2} \ddot{R}_{c}}{2 c_{s}^{2}}\left(\frac{R_{c 0}^{2}}{r^{2}}-1\right) .
\end{gathered}
$$

Note that the compressibility corrections are of the order of $1 / c_{s}^{2}$. Terms of the order $1 / c_{s}$ are absent. This means that with an accuracy up to the order $1 / c_{s}$, the solid can be approximated 
as an incompressible medium because there is no difference between solutions given by the compressible and incompressible equations of motion within the aforesaid accuracy.

\section{APPENDIX C: DERIVATION OF EQUATIONS FOR $R_{j}$ AND $z_{j}$}

Equations for $R_{j}$ and $z_{j}$ are derived by the method of the Lagrangian formalism. According to this method, we need to calculate the Lagrangian function $L=T-U$, where $T$ and $U$ are the kinetic and the potential energies of the system under study.

The kinetic energy of the liquid within the cavity is given by

$$
T_{l}=\frac{\rho_{l}}{2} \int_{V_{l}}(\nabla \varphi)^{2} d V_{l},
$$

where $\rho_{l}$ is the liquid density and $V_{l}$ is the volume occupied by the liquid. Equation $(\mathrm{C} 1)$ can be transformed as follows:

$$
\begin{gathered}
T_{l}=\frac{\rho_{l}}{2} \int_{V_{l}} \nabla \cdot(\varphi \nabla \varphi) d V_{l}=-\frac{\rho_{l}}{2}\left(\int_{S_{c}} \boldsymbol{n}_{c} \cdot \varphi \nabla \varphi d S_{c}+\int_{S_{1}} \boldsymbol{n}_{1} \cdot \varphi \nabla \varphi d S_{1}+\int_{S_{2}} \boldsymbol{n}_{2} \cdot \varphi \nabla \varphi d S_{2}\right) \\
=-\frac{\rho_{l}}{2}\left(\int_{S_{c}} \varphi \dot{R}_{c} d S_{c}+\int_{S_{1}} \varphi\left(\dot{R}_{1}+\dot{z}_{1} \cos \theta_{1}\right) d S_{1}+\int_{S_{2}} \varphi\left(\dot{R}_{2}+\dot{z}_{2} \cos \theta_{2}\right) d S_{2}\right)
\end{gathered}
$$

where $S_{c}$ and $S_{j}$ denote the surfaces of the cavity and of the $j$ th bubble, respectively, and $\boldsymbol{n}_{c}$ and $\boldsymbol{n}_{j}$ are the unit vectors normal to the surfaces of the cavity and of the $j$ th bubble, respectively, and directed into the liquid. When transforming Eq. (C1), we have used the Laplace equation $\Delta \varphi=0$ to replace $(\nabla \varphi)^{2}$ with $\nabla \cdot(\varphi \nabla \varphi)$ and Gauss's theorem to go from the volume integral to the surface integrals [32].

On substitution of the expressions for $\varphi$ near the cavity surface and near the bubble surfaces, Eq. (C2) gives

$$
\begin{gathered}
T_{l}=2 \pi \rho_{l}\left[R_{c 0}^{3} \dot{R}_{c}^{2}+R_{1}^{3}\left(\dot{R}_{1}^{2}+\frac{\dot{z}_{1}^{2}}{6}\right)+R_{2}^{3}\left(\dot{R}_{2}^{2}+\frac{\dot{z}_{2}^{2}}{6}\right)\right] \\
-\pi \rho_{l} R_{1}^{2}\left[2 \dot{R}_{1}\left(b_{0}^{(2)}+A_{0}^{(1)}\right)+\dot{z}_{1}\left(\xi_{1} b_{1}^{(2)}+A_{1}^{(1)}\right)\right]-\pi \rho_{l} R_{2}^{2}\left[2 \dot{R}_{2}\left(b_{0}^{(1)}+A_{0}^{(2)}\right)+\dot{z}_{2}\left(\xi_{2} b_{1}^{(1)}+A_{1}^{(2)}\right)\right] .
\end{gathered}
$$

In Eq. (C3), we have used Eqs. (A14), (A15), and (A17).

The potential energy of the liquid is calculated by 


$$
U_{l}=-\frac{4 \pi}{3} R_{1}^{3} P_{1}(t)-\frac{4 \pi}{3} R_{2}^{3} P_{2}(t)-z_{1} F_{D 1}-z_{2} F_{D 2},
$$

where $P_{j}(t)$ is the scattered pressure at the surface of the $j$ th bubble and $F_{D j}$ is the viscous drag force on the $j$ th bubble. It should be noted that the potential energy itself is given by the first two terms of Eq. (C4). The inclusion of the last two terms is an ad-hoc way that allows one to include the viscous drag forces in the equations of translational motion of the bubbles [22,25,29,33,34].

To allow for liquid compressibility corrections, the pressure $P_{j}$ can be taken in the form proposed by van der Meer et al. [35],

$$
P_{j}=P_{g j}\left(\frac{R_{j 0}}{R_{j}}\right)^{3 \gamma}\left(1-\frac{3 \gamma \dot{R}_{j}}{c_{l}}\right)-\frac{4 \eta_{l} \dot{R}_{j}}{R_{j}}-\frac{2 \sigma_{l}}{R_{j}}-P_{0}-P_{a c},
$$

where $P_{g j}=P_{0}+2 \sigma_{l} / R_{j 0}$ is the equilibrium gas pressure inside the $j$ th bubble, $R_{j 0}$ is the equilibrium radius of the $j$ th bubble, $\gamma$ is the ratio of specific heats of the gas, $c_{l}$ is the speed of sound in the liquid, $\eta_{l}$ is the dynamic viscosity of the liquid, $\sigma_{l}$ is the surface tension, $P_{0}$ is the hydrostatic pressure in the liquid, and $P_{a c}$ is the driving acoustic pressure.

If viscous effects are restricted to the thin boundary layer at the bubble surfaces, the viscous drag force is shown in Ref. [36] to be given by

$$
F_{D j}=-12 \pi \eta_{l} R_{j} \dot{z}_{j} .
$$

According to the analysis performed in Ref. [36], Eq. (C6) is valid not only in the limit of high Reynolds numbers but also for moderate or even low Reynolds numbers, provided the condition $R_{j} \dot{R}_{j} \rho_{l} / \eta_{l}>>1$ is satisfied.

The kinetic energy of the solid, making use of Eq. (9) and keeping up to $1 / c_{s}^{2}$, is calculated by

$$
T_{s}=\frac{\rho_{s}}{2} \int_{V_{s}}\left(\frac{\partial u}{\partial t}\right)^{2} d V_{s}=2 \pi \rho_{s} R_{c 0}^{3}\left(1-\frac{R_{c 0}}{R_{s}}\right)\left[\dot{R}_{c}^{2}-\frac{\dot{R}_{c} \dddot{R}_{c} R_{c 0} R_{s}}{c_{s}^{2}}\left(1-\frac{R_{c 0}}{R_{s}}\right)\right]
$$

where $V_{s}$ denotes the volume occupied by the solid and $R_{s}$ is the external radius of the solid layer.

The potential energy of the solid is calculated by

$$
U_{s}=\int_{V_{s}} \varepsilon d V_{s}
$$


where $\varepsilon$ denotes the elastic energy density, which is defined by [21]

$$
\varepsilon=\mu\left(u_{i j}-\frac{1}{3} \delta_{i j} u_{k k}\right)^{2}+\frac{1}{2} K u_{k k}^{2}=\mu\left[\left(\frac{\partial u}{\partial r}\right)^{2}+\frac{2 u^{2}}{r^{2}}\right]+\left(\frac{1}{2} K-\frac{1}{3} \mu\right)\left(\Delta \varphi_{s}\right)^{2} .
$$

Here $u_{i j}$ is the linear strain tensor, $\mu$ is the shear modulus, $K$ is bulk modulus, and summation over double indices is implied. Substituting Eq. (8) into Eq. (C9) and keeping up to $1 / c_{s}^{2}$, one obtains

$$
\varepsilon=\frac{6 \mu R_{c 0}^{4}\left(R_{c}-R_{c 0}\right)^{2}}{r^{6}}+\frac{2 \mu R_{c 0}^{4} \ddot{R}_{c}\left(R_{c}-R_{c 0}\right)}{c_{s}^{2} r^{4}}\left(\frac{3 R_{c 0}^{2}}{r^{2}}-1\right) .
$$

Substitution of Eq. (C10) into Eq. (C8) yields

$$
U_{s}=8 \pi \mu R_{c 0}\left(R_{c}-R_{c 0}\right)\left[\left(R_{c}-R_{c 0}\right)\left(1-\frac{R_{c 0}^{3}}{R_{s}^{3}}\right)+\frac{R_{c 0}^{3} \ddot{R}_{c}}{c_{s}^{2} R_{s}}\left(1-\frac{R_{c 0}^{2}}{R_{s}^{2}}\right)\right] .
$$

Let us return to Eq. (C3). It gives the kinetic energy of the liquid $T_{l}$ in terms of the functions $b_{0}^{(j)}, b_{1}^{(j)}, A_{0}^{(j)}$, and $A_{1}^{(j)}$. These functions can be calculated as expansions in series, considering $\xi_{j}, R_{j} / R_{c 0}$, and $z_{j} / R_{c 0}$ as expansion parameters because these quantities are always smaller than unity. We will carry out this calculation with an accuracy up to terms of third order of smallness.

From Eqs. (A22) and (A23), it follows that

$$
\begin{gathered}
a_{0}^{(1)}=-R_{1} \dot{R}_{1}, \\
a_{1}^{(1)}=-\frac{1}{2} R_{1} \dot{z}_{1}+\frac{1}{2} \xi_{1} \xi_{2} a_{0}^{(2)}-\xi_{1} \xi_{2}^{2} a_{1}^{(2)}+\frac{R_{1}}{R_{c 0}^{3}}\left(R_{1} z_{1} a_{0}^{(1)}+R_{2} z_{2} a_{0}^{(2)}+R_{1}^{2} a_{1}^{(1)}+R_{2}^{2} a_{1}^{(2)}\right), \\
a_{0}^{(2)}=-R_{2} \dot{R}_{2}, \\
a_{1}^{(2)}=-\frac{1}{2} R_{2} \dot{z}_{2}-\frac{1}{2} \xi_{1} \xi_{2} a_{0}^{(1)}-\xi_{1}^{2} \xi_{2} a_{1}^{(1)}+\frac{R_{2}}{R_{c 0}^{3}}\left(R_{1} z_{1} a_{0}^{(1)}+R_{2} z_{2} a_{0}^{(2)}+R_{1}^{2} a_{1}^{(1)}+R_{2}^{2} a_{1}^{(2)}\right) .
\end{gathered}
$$

From these equations, with an accuracy up to terms of third order, one obtains

$$
\begin{aligned}
& a_{1}^{(1)}=-\frac{1}{2} R_{1} \dot{z}_{1}\left(1+\frac{R_{1}^{3}}{R_{c 0}^{3}}\right)-\frac{1}{2} R_{2} \dot{R}_{2} \xi_{1} \xi_{2}+\frac{1}{2} R_{2} \dot{z}_{2}\left(\xi_{1} \xi_{2}^{2}-\frac{R_{1} R_{2}^{2}}{R_{c 0}^{3}}\right)-\frac{R_{1}^{3}}{R_{c 0}^{3}} \dot{R}_{1} z_{1}-\frac{R_{1} R_{2}^{2}}{R_{c 0}^{3}} \dot{R}_{2} z_{2}, \\
& a_{1}^{(2)}=-\frac{1}{2} R_{2} \dot{z}_{2}\left(1+\frac{R_{2}^{3}}{R_{c 0}^{3}}\right)+\frac{1}{2} R_{1} \dot{R}_{1} \xi_{1} \xi_{2}+\frac{1}{2} R_{1} \dot{z}_{1}\left(\xi_{1}^{2} \xi_{2}-\frac{R_{1}^{2} R_{2}}{R_{c 0}^{3}}\right)-\frac{R_{2}^{3}}{R_{c 0}^{3}} \dot{R}_{2} z_{2}-\frac{R_{1}^{2} R_{2}}{R_{c 0}^{3}} \dot{R}_{1} z_{1} .
\end{aligned}
$$

Substitution of Eqs. (C12), (C14), (C16), and (C17) into Eqs. (A5), (A6), and (A21) results in 


$$
\begin{gathered}
b_{0}^{(1)}=-R_{1} \dot{R}_{1} \xi_{1}-\frac{1}{2} R_{1} \dot{z}_{1} \xi_{1}^{2}, \\
b_{1}^{(1)}=R_{1} \dot{R}_{1} \xi_{1}+R_{1} \dot{z}_{1} \xi_{1}^{2}, \\
b_{0}^{(2)}=-R_{2} \dot{R}_{2} \xi_{2}+\frac{1}{2} R_{2} \dot{z}_{2} \xi_{2}^{2}, \\
b_{1}^{(2)}=-R_{2} \dot{R}_{2} \xi_{2}+R_{2} \dot{z}_{2} \xi_{2}^{2} . \\
A_{0}^{(j)}=-\frac{z_{j}}{R_{c 0}^{3}}\left(2 R_{1}^{2} \dot{R}_{1} z_{1}+2 R_{2}^{2} \dot{R}_{2} z_{2}+R_{1}^{3} \dot{z}_{1}+R_{2}^{3} \dot{z}_{2}\right), \\
A_{1}^{(j)}=-\frac{R_{j}}{R_{c 0}^{3}}\left(2 R_{1}^{2} \dot{R}_{1} z_{1}+2 R_{2}^{2} \dot{R}_{2} z_{2}+R_{1}^{3} \dot{z}_{1}+R_{2}^{3} \dot{z}_{2}\right) .
\end{gathered}
$$

Substitution of Eqs. (C18) - (C23) into Eq. (C3) yields

$$
\begin{gathered}
T_{l}=2 \pi \rho_{l}\left[R_{c 0}^{3} \dot{R}_{c}^{2}+R_{1}^{3}\left(\dot{R}_{1}^{2}+\frac{\dot{z}_{1}^{2}}{6}\right)+R_{2}^{3}\left(\dot{R}_{2}^{2}+\frac{\dot{z}_{2}^{2}}{6}\right)\right] \\
+2 \pi \rho_{l}\left[\frac{2 R_{1}^{2} R_{2}^{2} \dot{R}_{1} \dot{R}_{2}}{d}+\frac{R_{1}^{2} R_{2}^{2}\left(R_{1} \dot{R}_{2} \dot{z}_{1}-R_{2} \dot{R}_{1} \dot{z}_{2}\right)}{d^{2}}-\frac{R_{1}^{3} R_{2}^{3} \dot{z}_{1} \dot{z}_{2}}{d^{3}}\right] \\
+\frac{\pi \rho_{l}}{R_{c 0}^{3}}\left(4 R_{1}^{4} \dot{R}_{1}^{2} z_{1}^{2}+4 R_{1}^{5} \dot{R}_{1} z_{1} \dot{z}_{1}+R_{1}^{6} \dot{z}_{1}^{2}+4 R_{2}^{4} \dot{R}_{2}^{2} z_{2}^{2}+4 R_{2}^{5} \dot{R}_{2} z_{2} \dot{z}_{2}+R_{2}^{6} \dot{z}_{2}^{2}\right) \\
+\frac{2 \pi \rho_{l} R_{1}^{2} R_{2}^{2}}{R_{c 0}^{3}}\left(4 \dot{R}_{1} \dot{R}_{2} z_{1} z_{2}+2 R_{2} \dot{R}_{1} z_{1} \dot{z}_{2}+2 R_{1} \dot{R}_{2} \dot{z}_{1} z_{2}+R_{1} R_{2} \dot{z}_{1} \dot{z}_{2}\right) .
\end{gathered}
$$

We now can apply the Lagrangian equation,

$$
\frac{d}{d t} \frac{\partial L}{\partial \dot{q}_{j}}-\frac{\partial L}{\partial q_{j}}=0
$$

in which $R_{j}$ and $z_{j}$ should fulfil the role of the generalized coordinates $q_{j}$.

Note that, as follows from Eq. (A19), $\dot{R}_{c}$ is a function of $R_{j}$ and $\dot{R}_{j}$, so we can write

$$
\frac{\partial \dot{R}_{c}}{\partial R_{j}}=\frac{2 R_{j} \dot{R}_{j}}{R_{c 0}^{2}}, \quad \frac{\partial \dot{R}_{c}}{\partial \dot{R}_{j}}=\frac{R_{j}^{2}}{R_{c 0}^{2}} .
$$

In addition, the following equation is valid:

$$
R_{c}^{3}-R_{1}^{3}-R_{2}^{3}=R_{c 0}^{3}-R_{10}^{3}-R_{20}^{3},
$$

which gives 


$$
\frac{\partial R_{c}}{\partial R_{j}}=\frac{R_{j}^{2}}{R_{c}^{2}}
$$

Equations $(\mathrm{C} 26)-(\mathrm{C} 28)$ are used when the derivatives with respect to $R_{j}$ and $\dot{R}_{j}$ in Eq. (C25) are calculated.

Another point to be made is the appearance of $\dddot{R}_{c}$ in Eq. (C7) and $\ddot{R}_{c}$ in Eq. (C11). These quantities should be considered as time functions when Eqs. (C7) and (C11) are substituted into Eq. (C25).

With these remarks, substituting Eqs. (C4), (C7), (C11), and (C24) into Eq. (C25), one obtains

$$
\begin{gathered}
R_{1} \ddot{R}_{1}\left[1+F\left(R_{1}, z_{1}\right)\right]+\dot{R}_{1}^{2}\left[\frac{3}{2}+2 F\left(R_{1}, z_{1}\right)\right]-\frac{\dot{z}_{1}^{2}}{4}\left(1+\frac{2 R_{1}^{3}}{R_{c 0}^{3}}\right)-\frac{P_{1}}{\rho_{l}}+\frac{4 \mu}{\rho_{l}}\left(\frac{R_{c}}{R_{c 0}}-1\right)\left(1-\frac{R_{c 0}^{3}}{R_{s}^{3}}\right)-\frac{H}{c_{s}^{2}} \\
+\left(R_{2} \ddot{R}_{2}+2 \dot{R}_{2}^{2}\right) G\left(R_{2}, z_{1}, z_{2}\right)+\frac{R_{1}^{3} z_{1} \ddot{z}_{1}}{R_{c 0}^{3}}-R_{2} \ddot{z}_{2}\left(\frac{R_{2}^{2}}{2 d^{2}}-\frac{R_{2}^{2} z_{1}}{R_{c 0}^{3}}\right) \\
-\frac{R_{2}^{2} \dot{R}_{2}\left(\dot{z}_{1}+5 \dot{z}_{2}\right)}{2 d^{2}}+\frac{R_{2}^{3} \dot{z}_{2}\left(\dot{z}_{1}+2 \dot{z}_{2}\right)}{2 d^{3}}+\frac{8 R_{1}^{2} \dot{R}_{1} z_{1} \dot{z}_{1}+2 R_{2}^{2} \dot{R}_{2}\left(5 z_{1} \dot{z}_{2}-\dot{z}_{1} z_{2}\right)-R_{2}^{3} \dot{z}_{1} \dot{z}_{2}}{2 R_{c 0}^{3}}=0, \quad(\mathrm{C} 29) \\
R_{2} \ddot{R}_{2}\left[1+F\left(R_{2}, z_{2}\right)\right]+\dot{R}_{2}^{2}\left[\frac{3}{2}+2 F\left(R_{2}, z_{2}\right)\right]-\frac{\dot{z}_{2}^{2}}{4}\left(1+\frac{2 R_{2}^{3}}{R_{c 0}^{3}}\right)-\frac{P_{2}}{\rho_{l}}+\frac{4 \mu}{\rho_{l}}\left(\frac{R_{c}}{R_{c 0}}-1\right)\left(1-\frac{R_{c 0}^{3}}{R_{s}^{3}}\right)-\frac{H}{c_{s}^{2}} \\
+\left(R_{1} \ddot{R}_{1}+2 \dot{R}_{1}^{2}\right) G\left(R_{1}, z_{1}, z_{2}\right)+\frac{R_{2}^{3} z_{2} \ddot{z}_{2}}{R_{c 0}^{3}}+R_{1} \ddot{z}_{1}\left(\frac{R_{1}^{2}}{2 d^{2}}+\frac{R_{1}^{2} z_{2}}{R_{c 0}^{3}}\right) \\
+R_{2}^{2}\left[R_{1} R_{2} \ddot{z}_{2}+\dot{z}_{2}\left(R_{2} \dot{R}_{1}+5 R_{1} \dot{R}_{2}\right)\right]\left(\frac{1}{R_{c 0}^{3}}-\frac{1}{d^{3}}\right)=\frac{F_{D 1}}{2 \pi \rho_{l} R_{1}^{2}}, \\
+\frac{R_{1}^{2} \dot{R}_{1}\left(\dot{z}_{2}+5 \dot{z}_{1}\right)}{2 d^{2}}+\frac{R_{1}^{3} \dot{z}_{1}\left(\dot{z}_{2}+2 \dot{z}_{1}\right)}{2 d^{3}}+\frac{8 R_{2}^{2} \dot{R}_{2} z_{2} \dot{z}_{2}+2 R_{1}^{2} \dot{R}_{1}\left(5 \dot{z}_{1} z_{2}-z_{1} \dot{z}_{2}\right)-R_{1}^{3} \dot{z}_{1} \dot{z}_{2}}{2 R_{c 0}^{3}}=0 \\
\left.\left.\frac{R_{1} \ddot{z}_{1}}{3}\left(1+\frac{3 R_{1}^{3}}{R_{c 0}^{3}}\right)+\dot{R}_{1} \dot{z}_{1}\left(1+\frac{6 R_{1}^{3}}{R_{c 0}^{3}}\right)+\frac{2 R_{1}^{2} z_{1}\left(R_{1} \ddot{R}_{1}+3 \dot{R}_{1}^{2}\right)}{R_{c 0}^{3}}+2 \dot{R}_{2}^{2}\right)+R_{2} \dot{R}_{1} \dot{R}_{2}\right]\left(\frac{2 z_{2}}{R_{c 0}^{3}}+\frac{1}{d^{2}}\right) \\
(\mathrm{C} 30)
\end{gathered}
$$




$$
\begin{gathered}
\frac{R_{2} \ddot{z}_{2}}{3}\left(1+\frac{3 R_{2}^{3}}{R_{c 0}^{3}}\right)+\dot{R}_{2} \dot{z}_{2}\left(1+\frac{6 R_{2}^{3}}{R_{c 0}^{3}}\right)+\frac{2 R_{2}^{2} z_{2}\left(R_{2} \ddot{R}_{2}+3 \dot{R}_{2}^{2}\right)}{R_{c 0}^{3}} \\
R_{1}\left[R_{2}\left(R_{1} \ddot{R}_{1}+2 \dot{R}_{1}^{2}\right)+R_{1} \dot{R}_{1} \dot{R}_{2}\right]\left(\frac{2 z_{1}}{R_{c 0}^{3}}-\frac{1}{d^{2}}\right) \\
+R_{1}^{2}\left[R_{1} R_{2} \ddot{z}_{1}+\dot{z}_{1}\left(R_{1} \dot{R}_{2}+5 R_{2} \dot{R}_{1}\right)\right]\left(\frac{1}{R_{c 0}^{3}}-\frac{1}{d^{3}}\right)=\frac{F_{D 2}}{2 \pi \rho_{l} R_{2}^{2}},
\end{gathered}
$$

where

$$
\begin{gathered}
F\left(R_{j}, z_{j}\right)=\frac{R_{j}}{R_{c 0}}\left[1+\frac{\rho_{s}}{\rho_{l}}\left(1-\frac{R_{c 0}}{R_{s}}\right)\right]+\frac{2 R_{j} z_{j}^{2}}{R_{c 0}^{3}}+\frac{2 \mu R_{j}}{c_{s}^{2} \rho_{l} R_{s}}\left(1-\frac{R_{c 0}^{2}}{R_{s}^{2}}\right) \\
G\left(R_{j}, z_{1}, z_{2}\right)=\frac{R_{j}}{d}+\frac{R_{j}}{R_{c 0}}\left[1+\frac{\rho_{s}}{\rho_{l}}\left(1-\frac{R_{c 0}}{R_{s}}\right)\right]+\frac{2 R_{j} z_{1} z_{2}}{R_{c 0}^{3}}+\frac{2 \mu R_{j}}{c_{s}^{2} \rho_{l} R_{s}}\left(1-\frac{R_{c 0}^{2}}{R_{s}^{2}}\right), \\
H=\frac{R_{c 0}^{2} R_{s} \rho_{s}}{2 \rho_{l}}\left(1-\frac{R_{c 0}}{R_{s}}\right)^{2} \frac{d^{4} R_{c}}{d t^{4}} .
\end{gathered}
$$

As one can see, Eqs. (C29) and (C30) involve $d^{4} R_{c} / d t^{4}$, which appears in a correction term that is caused by the compressibility of the solid. Since this term is of the order $1 / c_{s}^{2}$, it will suffice to evaluate $d^{4} R_{c} / d t^{4}$ with an accuracy up to leading terms, neglecting all secondary effects. To this end, we can apply the following approximation.

From Eq. (A19), it follows that

$$
R_{c 0}^{2} \ddot{R}_{c}=R_{1}^{2} \ddot{R}_{1}+2 R_{1} \dot{R}_{1}^{2}+R_{2}^{2} \ddot{R}_{2}+2 R_{2} \dot{R}_{2}^{2} .
$$

We can express $\ddot{R}_{j}$ in terms of $\dot{R}_{j}$ by the Rayleigh-Plesset equation for a single bubble in an unbounded liquid,

$$
R_{j}^{2} \ddot{R}_{j}=-\frac{3}{2} R_{j} \dot{R}_{j}^{2}+\frac{R_{j} P_{j}}{\rho_{l}}
$$

Substitution of Eq. (C37) into Eq. (C36) yields

$$
R_{c 0}^{2} \ddot{R}_{c}=\frac{1}{2} R_{1} \dot{R}_{1}^{2}+\frac{1}{2} R_{2} \dot{R}_{2}^{2}+\frac{1}{\rho_{l}}\left(R_{1} P_{1}+R_{2} P_{2}\right) .
$$

Differentiation of this equation with respect to time gives 


$$
R_{c 0}^{2} \dddot{R_{c}}=R_{1} \dot{R}_{1} \ddot{R}_{1}+R_{2} \dot{R}_{2} \ddot{R}_{2}+\frac{1}{2} \dot{R}_{1}^{3}+\frac{1}{2} \dot{R}_{2}^{3}+\frac{1}{\rho_{l}}\left(\dot{R}_{1} P_{1}+R_{1} \dot{P}_{1}+\dot{R}_{2} P_{2}+R_{2} \dot{P}_{2}\right) .
$$

Substituting Eq. (C37) for $\ddot{R}_{j}$, one obtains

$$
R_{c 0}^{2} \dddot{R}_{c}=-\dot{R}_{1}^{3}-\dot{R}_{2}^{3}+\frac{1}{\rho_{l}}\left(2 \dot{R}_{1} P_{1}+R_{1} \dot{P}_{1}+2 \dot{R}_{2} P_{2}+R_{2} \dot{P}_{2}\right) .
$$

Differentiation of Eq. (C40) with respect to time gives

$$
R_{c 0}^{2} \frac{d^{4} R_{c}}{d t^{4}}=\ddot{R}_{1}\left(\frac{2 P_{1}}{\rho_{l}}-3 \dot{R}_{1}^{2}\right)+\ddot{R}_{2}\left(\frac{2 P_{2}}{\rho_{l}}-3 \dot{R}_{2}^{2}\right)+\frac{1}{\rho_{l}}\left(3 \dot{R}_{1} \dot{P}_{1}+R_{1} \ddot{P}_{1}+3 \dot{R}_{2} \dot{P}_{2}+R_{2} \ddot{P}_{2}\right) .
$$

Expressions for $\dot{P}_{j}$ and $\ddot{P}_{j}$ should be calculated up to leading terms as well. In this approximation, Eq. (C5) gives

$$
\begin{gathered}
\dot{P}_{j}=\frac{\dot{R}_{j}}{R_{j}}\left[\frac{2 \sigma_{l}}{R_{j}}-3 \gamma P_{g j}\left(\frac{R_{j 0}}{R_{j}}\right)^{3 \gamma}\right]-\dot{P}_{a c}, \\
\ddot{P}_{j}=\frac{\ddot{R}_{j}}{R_{j}}\left[\frac{2 \sigma_{l}}{R_{j}}-3 \gamma P_{g j}\left(\frac{R_{j 0}}{R_{j}}\right)^{3 \gamma}\right]+\frac{\dot{R}_{j}^{2}}{R_{j}^{2}}\left[3 \gamma(3 \gamma+1) P_{g j}\left(\frac{R_{j 0}}{R_{j}}\right)^{3 \gamma}-\frac{4 \sigma_{l}}{R_{j}}\right]-\ddot{P}_{a c} .
\end{gathered}
$$

Substituting Eqs. (C42) and (C43) into Eq. (C41) and then substituting the result into Eq. (C35), one obtains

$$
H=\frac{R_{s} \rho_{s}\left(Q_{1}+Q_{2}\right)}{2 \rho_{l}}\left(1-\frac{R_{c 0}}{R_{s}}\right)^{2}
$$

where

$$
\begin{gathered}
Q_{j}=\frac{\ddot{R}_{j}}{\rho_{l}}\left[\frac{2 \sigma_{l}}{R_{j}}-3 \gamma P_{g j}\left(\frac{R_{j 0}}{R_{j}}\right)^{3 \gamma}+2 P_{j}-3 \rho_{l} \dot{R}_{j}^{2}\right] \\
+\frac{\dot{R}_{j}^{2}}{\rho_{l} R_{j}}\left[\frac{2 \sigma_{l}}{R_{j}}+3 \gamma(3 \gamma-2) P_{g j}\left(\frac{R_{j 0}}{R_{j}}\right)^{3 \gamma}\right]-\frac{3 \dot{R}_{j} \dot{P}_{a c}+R_{j} \ddot{a}_{a c}}{\rho_{l}} .
\end{gathered}
$$

Substitution of Eq. (C44) into Eqs. (C29) and (C30) makes them ordinary differential equations of second order with respect to the time derivatives of $R_{j}$. 
[1] E. Roedder and R. J. Bodnar, Annu. Rev. Earth Planet. Sci. 8, 263 (1980).

[2] D. Marti, Y. Krüger, D. Fleitmann, M. Frenz, and J. Rička, Fluid Phase Equilib. 314, 13 (2012).

[3] G. W. Scherer and D. M. Smith, J. Non-Cryst. Solids 189, 197 (1995).

[4] D. Or and M. Tuller, Water Resour. Res. 38, 1061 (2002).

[5] O. Vincent, D. A. Sessoms, E. J. Huber, J. Guioth, and A. D. Stroock, Phys. Rev. Lett. 113, 134501 (2014).

[6] M. T. Tyree and M. A. Dixon, Plant Physiol. 72, 1094 (1983).

[7] H. Cochard, C. R. Phys. 7, 1018 (2006).

[8] X. Noblin, N. O. Rojas, J. Westbrook, C. Llorens, M. Argentina, and J. Dumais, Science 335, 1322 (2012).

[9] A. D. Stroock, V. V. Pagay, M. A. Zwieniecki, and N. M. Holbrook, Annu. Rev. Fluid Mech. 46, 615 (2014).

[10] K. H. Jensen, H. Bruus, N. M. Holbrook, J. Liesche, A. Schulz, and M. A. Zwieniecki, Rev. Mod. Phys. 88, 035007 (2016).

[11] O. Vincent, P. Marmottant, P. A. Quinto-Su, and C.-D. Ohl, Phys. Rev. Lett. 108, 184502 (2012).

[12] O. Vincent, P. Marmottant, S. R. Gonzalez-Avila, K. Ando, and C.-D. Ohl, Soft Matter 10, 1455 (2014).

[13] O. Vincent and P. Marmottant, J. Fluid Mech. 827, 194 (2017).

[14] Q. X. Wang, Phys. Fluids 29, 072101 (2017).

[15] C. Drysdale, A. A. Doinikov, and P. Marmottant, Phys. Rev. E 95, 053104 (2017).

[16] A. A. Doinikov, B. Dollet, and P. Marmottant, Phys. Rev. E 97, 013108 (2018).

[17] V. F. K. Bjerknes, Fields of Force (Columbia University Press, New York, 1906).

[18] H. N. Oguz and A. Prosperetti, J. Fluid Mech. 218, 143 (1990).

[19] N. A. Pelekasis and J. A. Tsamopoulos, J. Fluid Mech. 254, 501 (1993).

[20] R. Mettin, I. Akhatov, U. Parlitz, C. D. Ohl, and W. Lauterborn, Phys. Rev. E 56, 2924 (1997).

[21] A. Harkin, T. J. Kaper, and A. Nadim, J. Fluid Mech. 445, 377 (2001).

[22] A. A. Doinikov, Phys. Rev. E 64, 026301 (2001).

[23] M. Ida, Phys. Lett. A 297, 210 (2002).

[24] J. S. Allen, D. E. Kruse, P. A. Dayton, and K. W. Ferrara, J. Acoust. Soc. Am. 114, 1678 (2003). 
[25] A. A. Doinikov, in Bubble and Particle Dynamics in Acoustic Fields: Modern Trends and Applications, edited by A. A. Doinikov (Research Signpost, Kerala, 2005), p. 95.

[26] A. A. Doinikov and A. Bouakaz, Phys. Rev. E 92, 043001 (2015).

[27] L. D. Landau and E. M. Lifshitz, Theory of Elasticity (Pergamon Press, Oxford, 1970).

[28] M. Minnaert, Phil. Mag. 16, 235 (1933).

[29] E. A. Zabolotskaya, Sov. Phys. Acoust. 30, 365 (1984).

[30] T. D. Wheeler and A. D. Stroock, Nature 45, 208 (2008).

[31] D. A. Varshalovich, A. N. Moskalev, and V. K. Khersonskii, Quantum Theory of Angular Momentum (Nauka, Moscow, 1975).

[32] G. Arfken, Mathematical Methods for Physicists (Academic Press, San Diego, 1985).

[33] S. Luther, R. Mettin, and W. Lauterborn, in Proceedings of the 15th International Symposium on Nonlinear Acoustics (AIP, Melville, NY, 2000), pp. 351-354.

[34] S. Y. Emelianov, M. F. Hamilton, Yu. A. Ilinskii, and E. A. Zabolotskaya, J. Acoust. Soc. Am. 115, 581 (2004).

[35] S. M. van der Meer, B. Dollet, M. M. Voormolen, C. T. Chin, A. Bouakaz, N. de Jong, M. Versluis, and D. Lohse, J. Acoust. Soc. Am. 121, 648 (2007).

[36] J. Magnaudet and D. Legendre, Phys. Fluids 10, 550 (1998). 


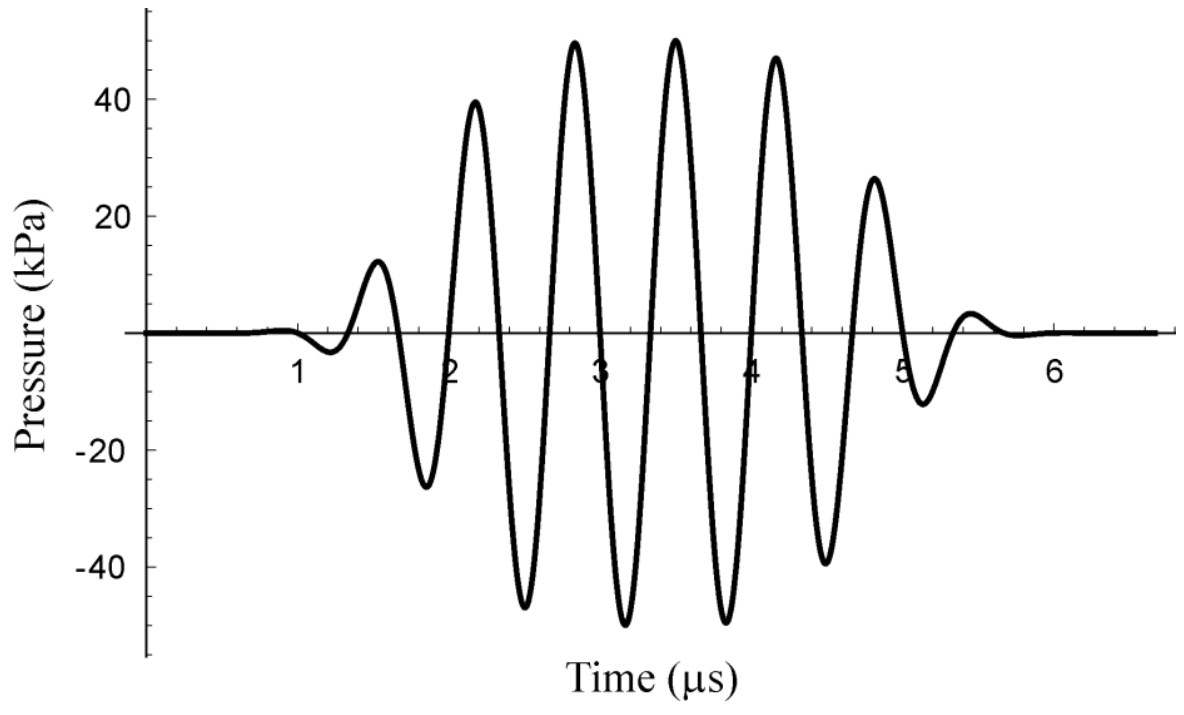

FIG. 2. Acoustic pulse used as excitation in Fig. 3. The mathematical definition is given by Eq. (32). The pulse parameters are $f=1.5 \mathrm{MHz}, P_{a}=50 \mathrm{kPa}$, and $N=5$. 

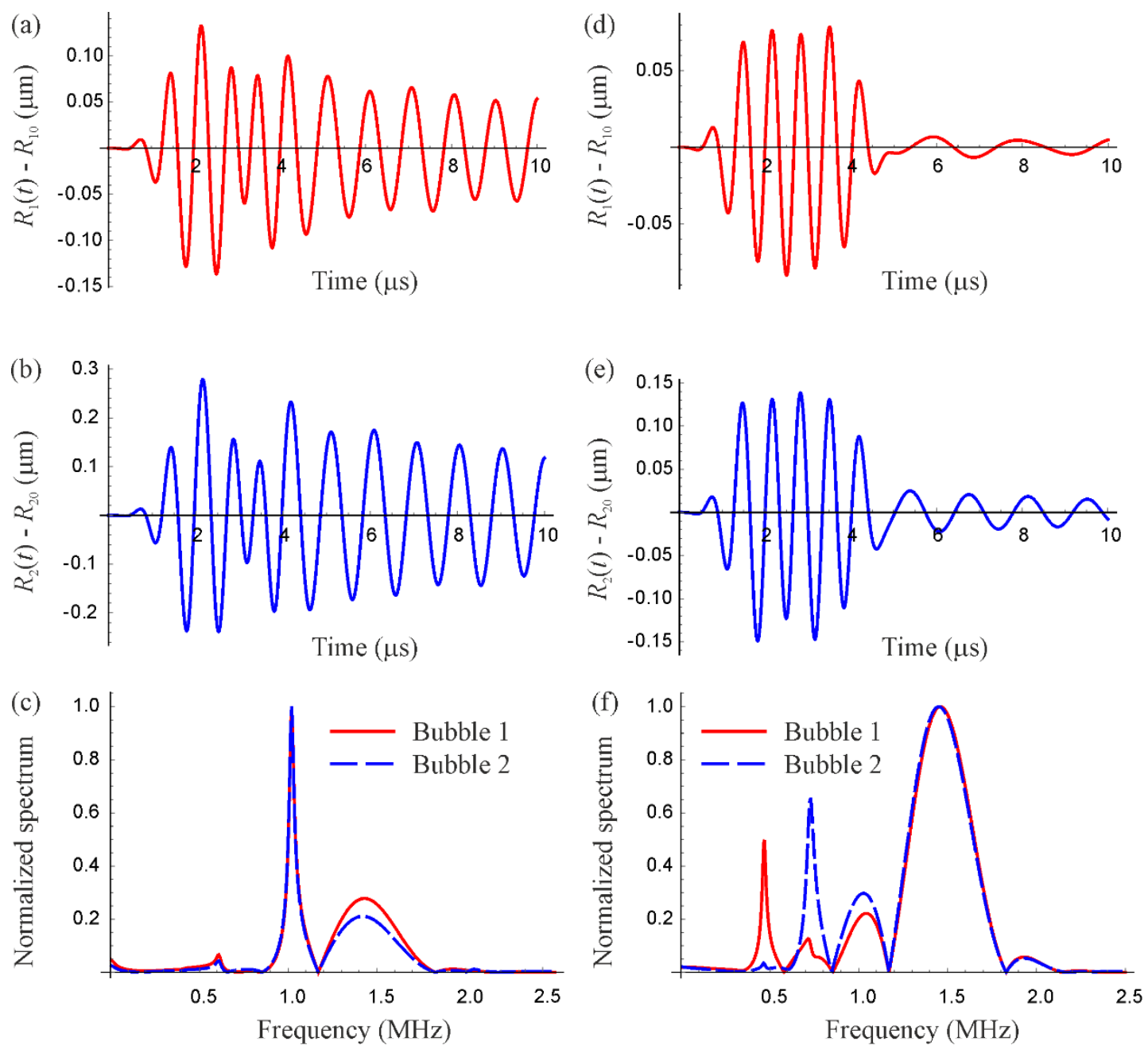

FIG. 3. Response of bubbles to the acoustic pulse shown in Fig. 2. Left panel: bubbles in a cavity. Right panel: bubbles in an unbounded liquid. $R_{10}=7.5 \mu \mathrm{m}, R_{20}=5 \mu \mathrm{m}, z_{1}(0)=-50 \mu \mathrm{m}$, $z_{2}(0)=50 \mu \mathrm{m}, R_{c 0}=100 \mu \mathrm{m}, R_{s}=500 \mu \mathrm{m}$. The Minnaert frequencies of the bubbles are 468 $\mathrm{kHz}$ and $724 \mathrm{kHz}$. The bubbles are excited above their natural frequencies. 


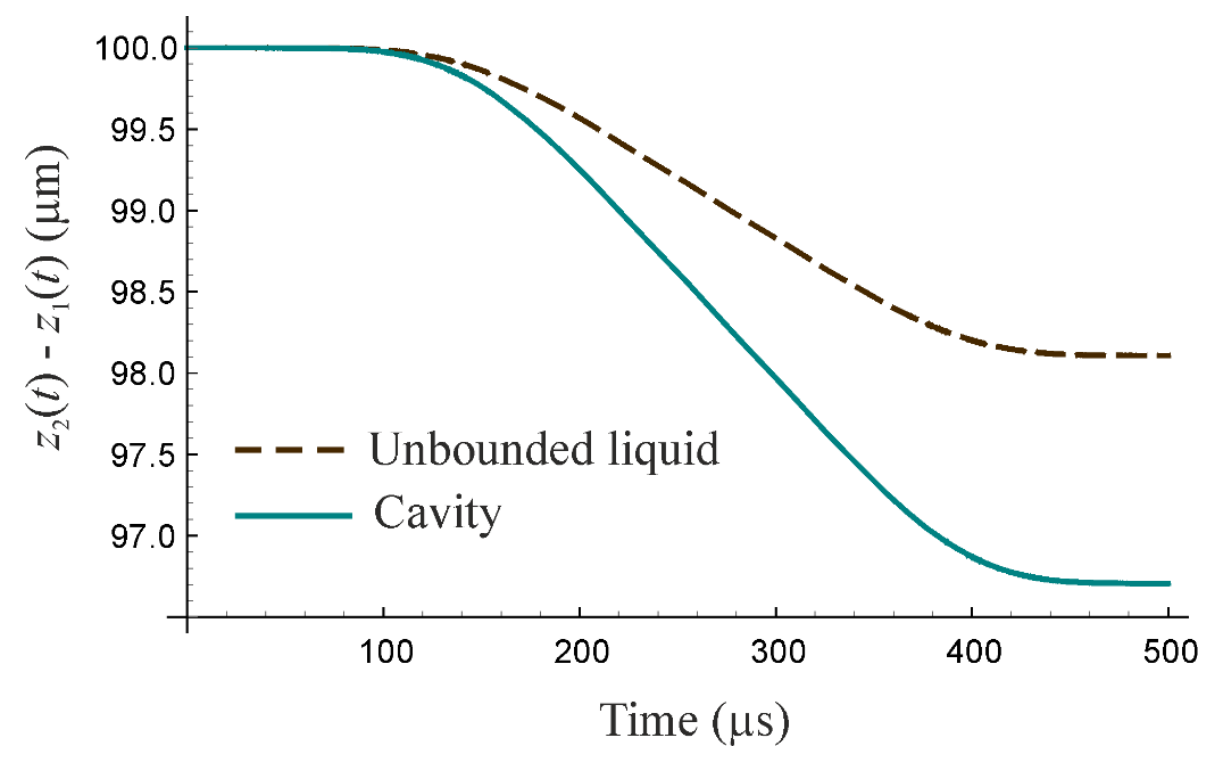

FIG. 4. Translational motion of two bubbles in a cavity and in an unbounded liquid. The parameters are as in Fig. 3, except that the number of cycles in the excitation pulse is $N=500$. The bubbles are moving toward each other. 

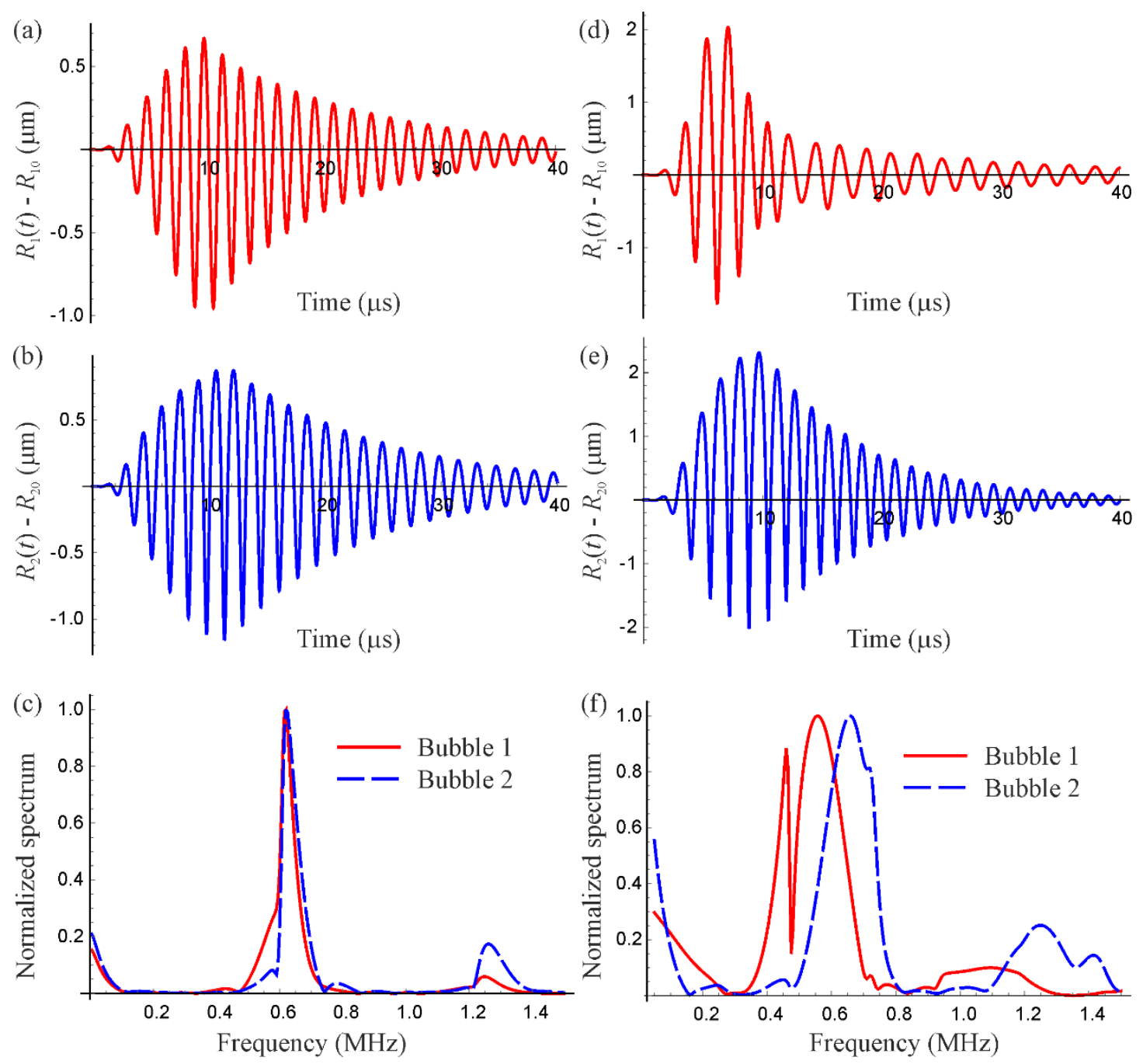

FIG. 5. Response of bubbles to an acoustic pulse with the parameters $f=0.6 \mathrm{MHz}, P_{a}=50 \mathrm{kPa}$, $N=5$. Left panel: bubbles in a cavity. Right panel: bubbles in an unbounded liquid. $R_{10}=7.5 \mu \mathrm{m}$, $R_{20}=5 \mu \mathrm{m}, z_{1}(0)=-50 \mu \mathrm{m}, z_{2}(0)=50 \mu \mathrm{m}, R_{c 0}=100 \mu \mathrm{m}, R_{s}=500 \mu \mathrm{m}$. The driving frequency lies between the natural frequencies of the bubbles. 


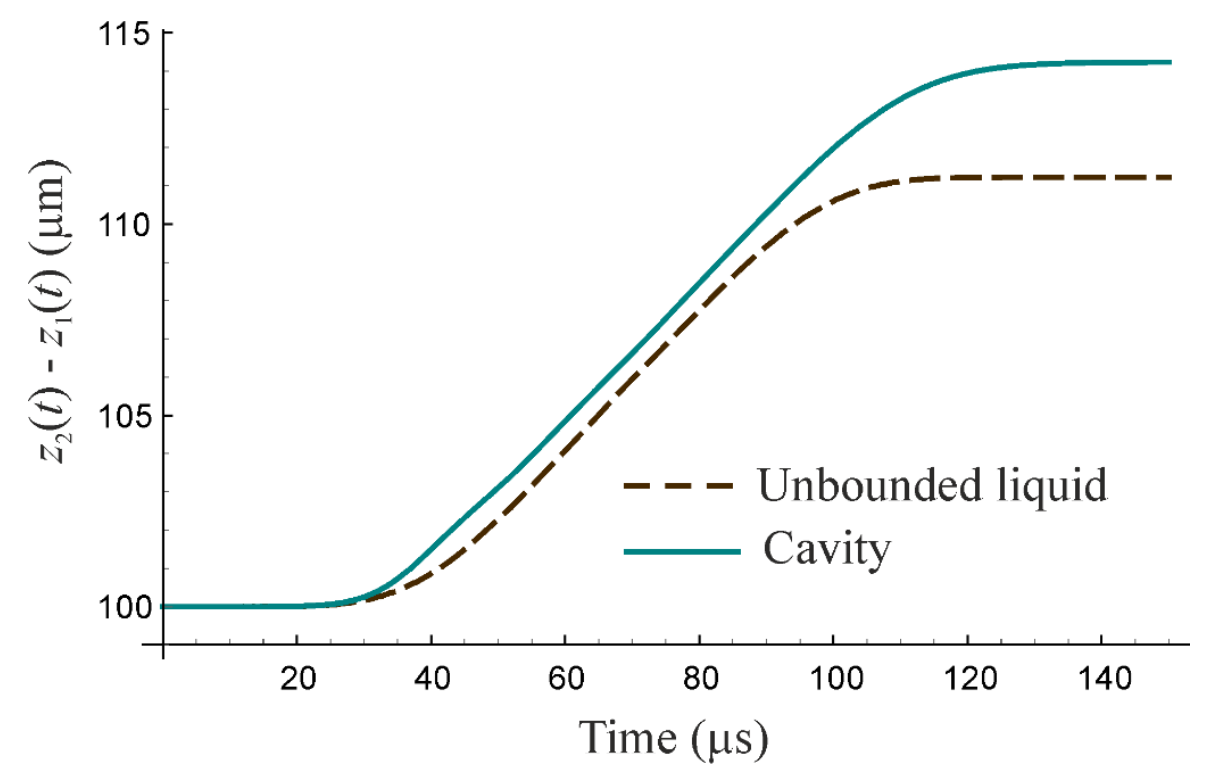

FIG. 6. Translational motion of two bubbles in a cavity and in an unbounded liquid. The parameters are as in Fig. 5, except that $N=50$. The bubbles are moving away from each other. 

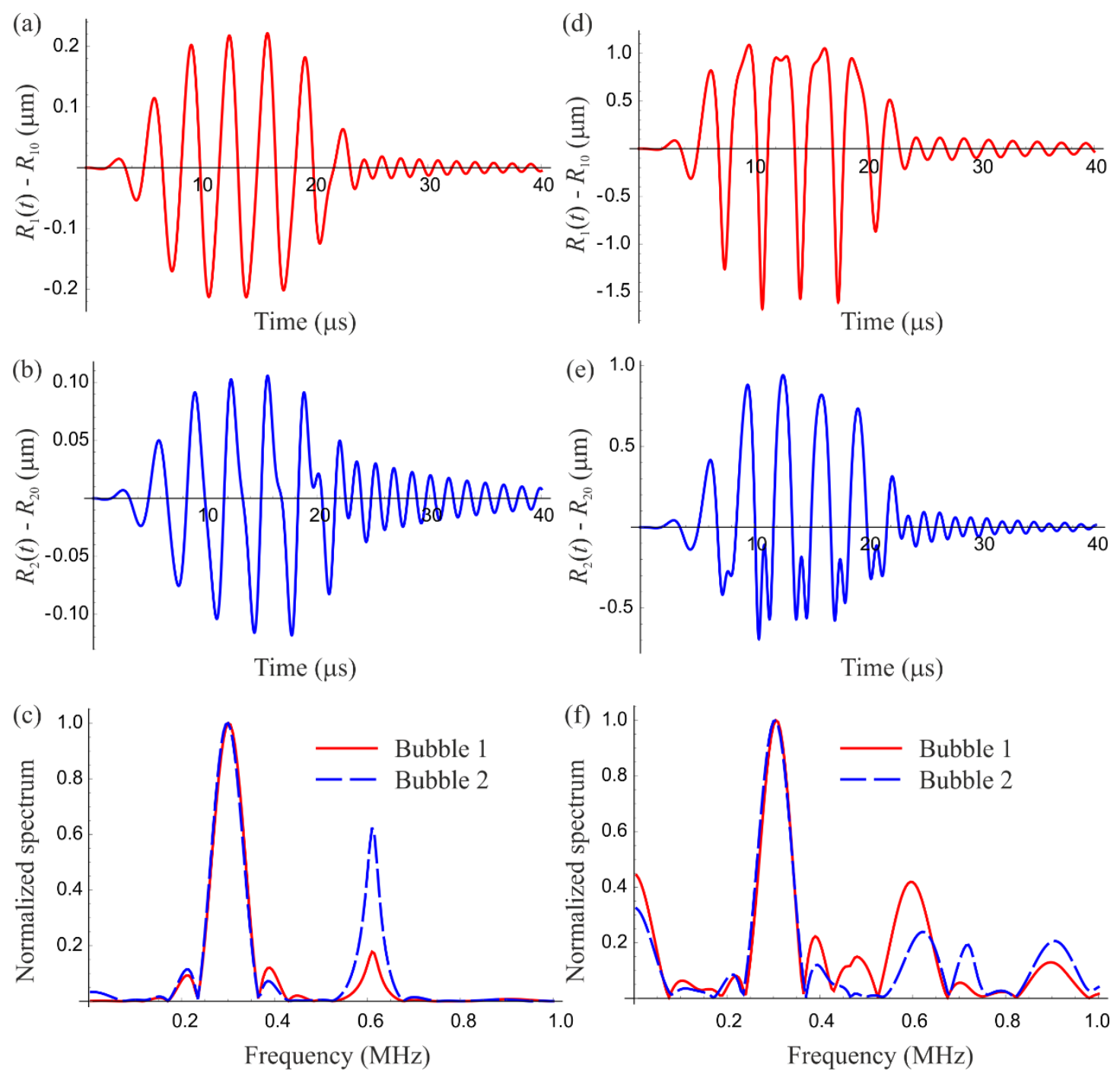

FIG. 7. Response of bubbles to an acoustic pulse with the parameters $f=0.3 \mathrm{MHz}, P_{a}=50 \mathrm{kPa}$, $N=5$. Left panel: bubbles in a cavity. Right panel: bubbles in an unbounded liquid. $R_{10}=7.5 \mu \mathrm{m}$, $R_{20}=5 \mu \mathrm{m}, z_{1}(0)=-50 \mu \mathrm{m}, z_{2}(0)=50 \mu \mathrm{m}, R_{c 0}=100 \mu \mathrm{m}, R_{s}=500 \mu \mathrm{m}$. The bubbles are excited below their natural frequencies. 


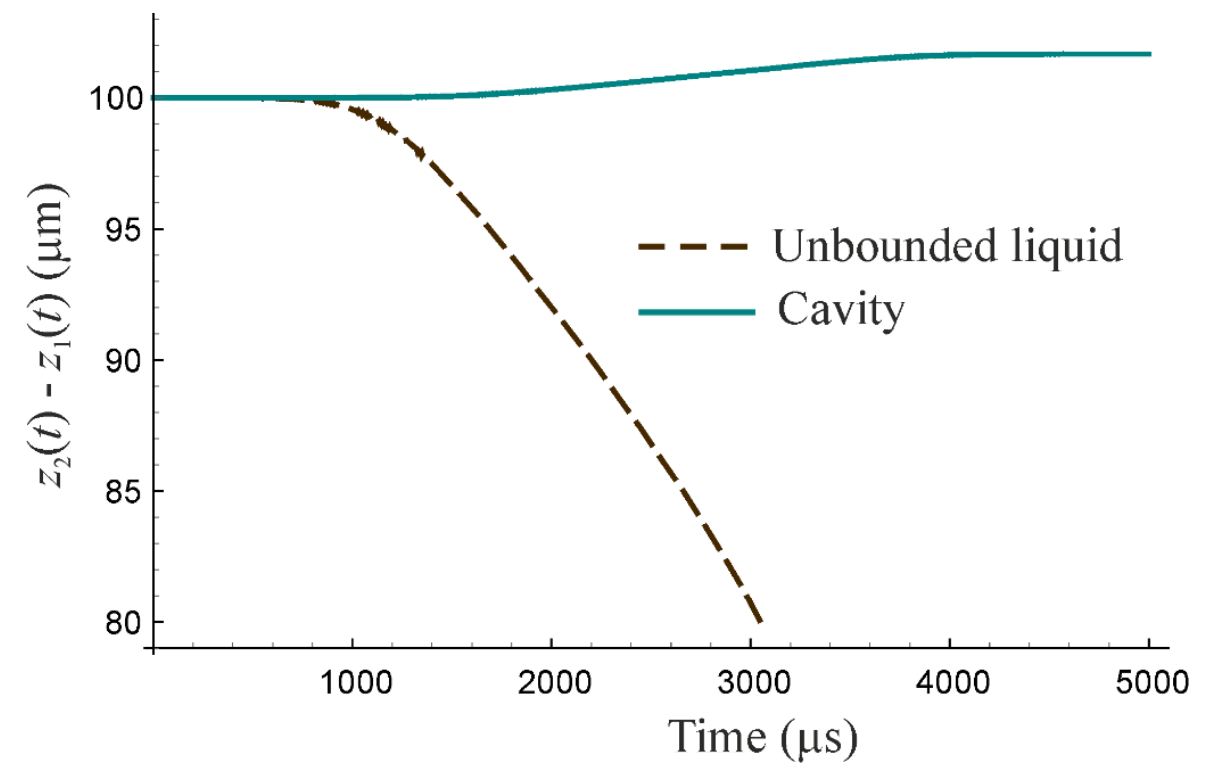

FIG. 8. Translational motion of two bubbles in a cavity and in an unbounded liquid. The parameters are as in Fig. 7, except that $N=1000$. 

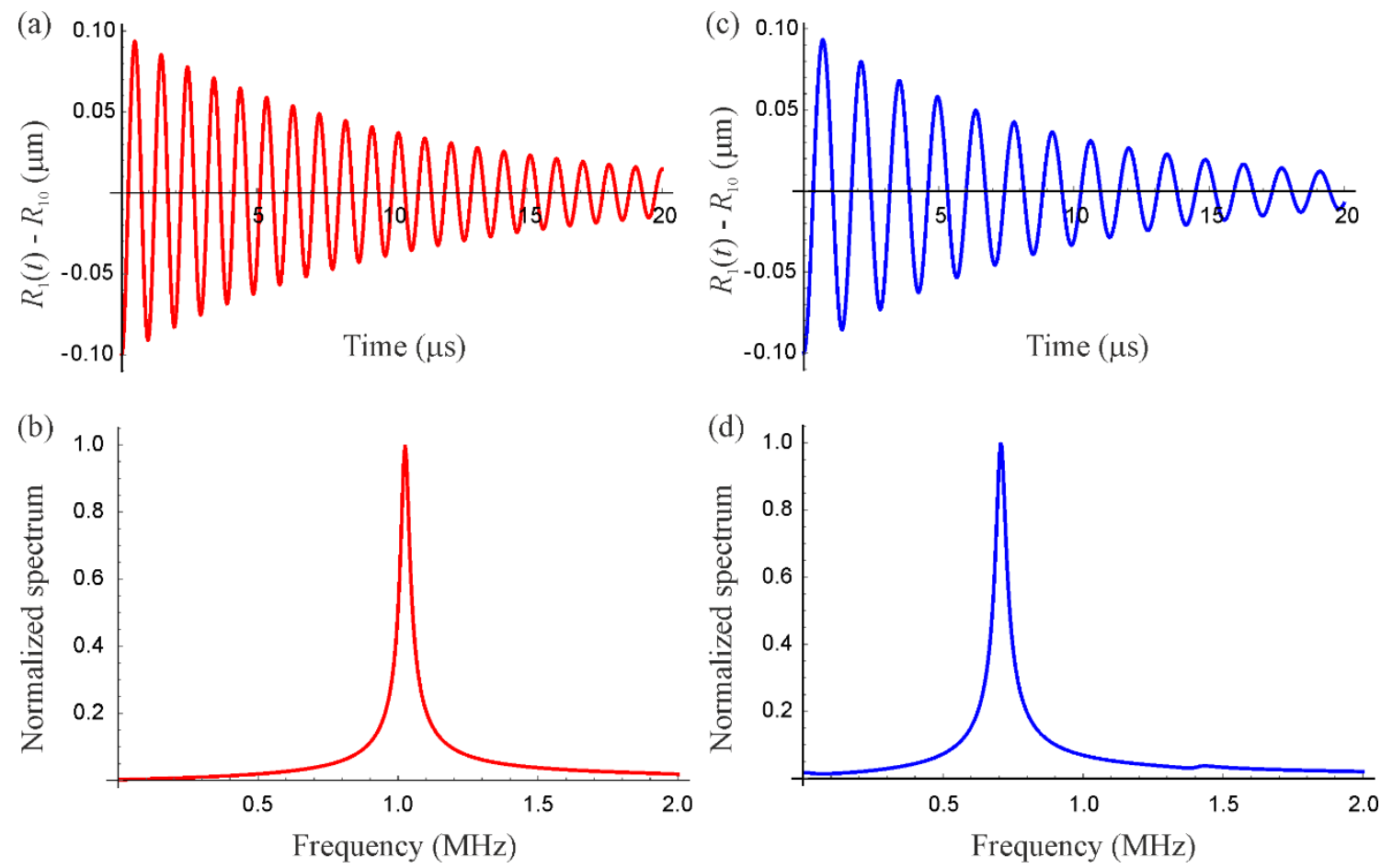

FIG. 9. Free oscillations of two equal bubbles. Left panel: bubbles in a cavity. Right panel: bubbles in an unbounded liquid. $R_{10}=R_{20}=5 \mu \mathrm{m}, z_{1}(0)=-50 \mu \mathrm{m}, z_{2}(0)=50 \mu \mathrm{m}, R_{c 0}=100 \mu \mathrm{m}$, $R_{s}=500 \mu \mathrm{m}$. The Minnaert frequency of the bubbles is $724 \mathrm{kHz}$. The free oscillations are excited by a small initial deviation from the equilibrium bubble radii. 

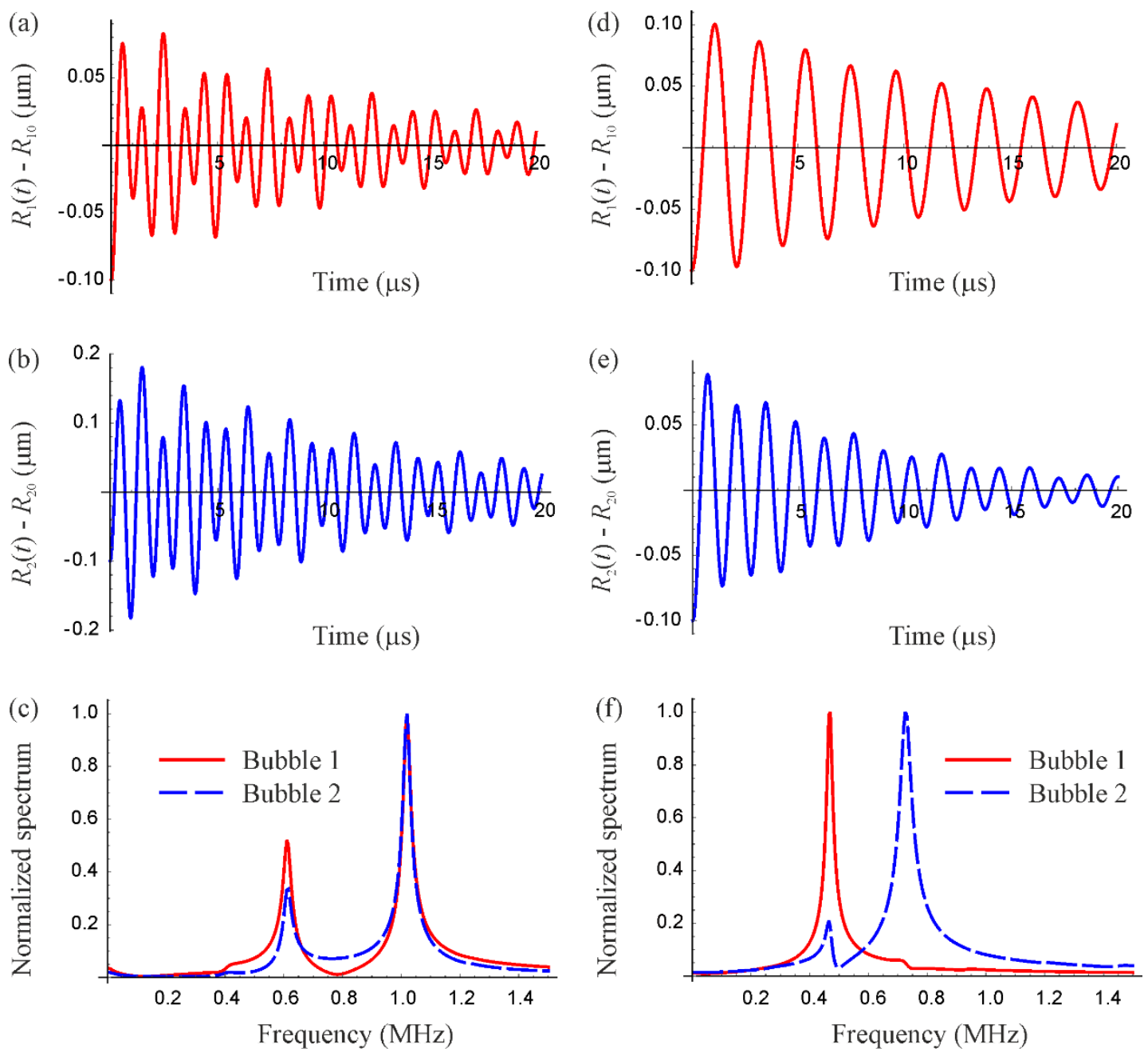

FIG. 10. Free oscillations of two unequal bubbles. Left panel: bubbles in a cavity. Right panel: bubbles in an unbounded liquid. $R_{10}=7.5 \mu \mathrm{m}, R_{20}=5 \mu \mathrm{m}, z_{1}(0)=-50 \mu \mathrm{m}, z_{2}(0)=50 \mu \mathrm{m}$, $R_{c 0}=100 \mu \mathrm{m}, R_{s}=500 \mu \mathrm{m}$. The Minnaert frequencies of the bubbles are $468 \mathrm{kHz}$ and $724 \mathrm{kHz}$. 


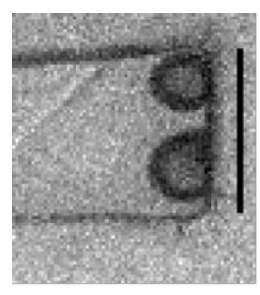

(a)

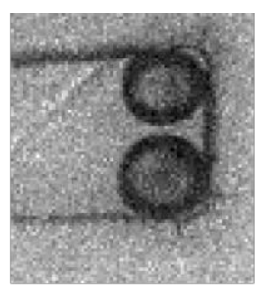

(b)

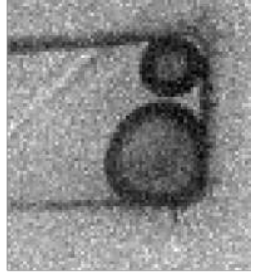

(c)

FIG. 11. Growth and evolution of two bubbles in a rectangular microchannel. Images are taken after nucleation at times (a) $1.9 \mu \mathrm{s}$, (b) $15 \mu \mathrm{s}$, (c) $45 \mu \mathrm{s}$. The vertical scale is $100 \mu \mathrm{m}$.

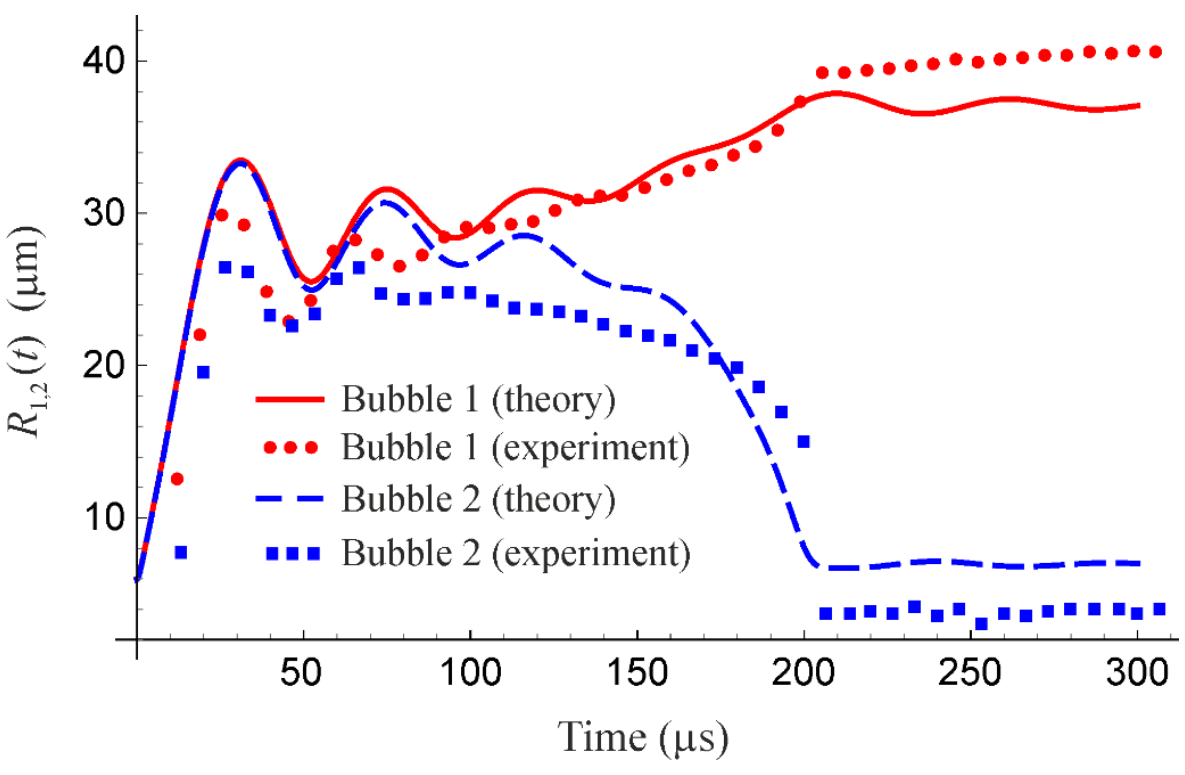

FIG. 12. Comparison of experimental data and theoretical predictions. 